\title{
CAPITULATION IN THE ABSOLUTELY ABELIAN EXTENSIONS OF SOME NUMBER FIELDS II
}

\author{
ABDELMALEK AZIZI, ABDELKADER ZEKHNINI, AND MOHAMMED TAOUS
}

\begin{abstract}
We study the capitulation of 2-ideal classes of an infinite family of imaginary biquadratic number fields consisting of fields $\mathbb{k}=\mathbb{Q}\left(\sqrt{p q_{1} q_{2}}, i\right)$, where $i=\sqrt{-1}$ and $q_{1} \equiv q_{2} \equiv-p \equiv-1(\bmod 4)$ are different primes. For each of the three quadratic extensions $\mathbb{K} / \mathbb{k}$ inside the absolute genus field $\mathbb{k}^{(*)}$ of $\mathrm{k}$, we compute the capitulation kernel of $\mathbb{K} / \mathbb{k}$. Then we deduce that each strongly ambiguous class of $\mathbb{k} / \mathbb{Q}(i)$ capitulates already in $\mathbb{k}^{(*)}$.
\end{abstract}

\section{Introduction and Notations}

Let $k$ be an algebraic number field and let $\mathbf{C} l_{2}(k)$ denote its 2-class group, that is the 2-Sylow subgroup of the ideal class group, $\mathbf{C} l(k)$, of $k$. We denote by $k^{(*)}$ the absolute genus field of $k$, that is the maximal abelian unramified extension of $k$ obtained by composing $k$ and an abelian extension over $\mathbb{Q}$.

Suppose $F$ is a finite extension of $k$, then we say that an ideal class of $k$ capitulates in $F$ if it is in the kernel of the homomorphism

$$
J_{F}: \mathbf{C l}(k) \longrightarrow \mathbf{C l}(F)
$$

induced by extension of ideals from $k$ to $F$. An important problem in Number Theory is to explicitly determine the kernel of $J_{F}$, which is usually called the capitulation kernel.

If $F$ is the relative genus field of a cyclic extension $K / k$, which we denote by $(K / k)^{*}$ and that is the maximal unramified extension of $K$ which is obtained by composing $K$ and an abelian extension over $k, \mathrm{~F}$. Terada states in [15] that all the ambiguous ideal classes of $K / k$, which are classes of $K$ fixed under any element of $\mathrm{Gal}(K / k)$, capitulate in $(K / k)^{*}$.

If $F$ is the absolute genus field of an abelian extension $K / \mathbb{Q}$, then $H$. Furuya confirms in [16] that every strongly ambiguous class of $K / \mathbb{Q}$, that is an ambiguous ideal class containing at least one ideal invariant under $\mathrm{Gal}(K / \mathbb{Q})$, capitulates in $F$.

In this paper, we construct a family of number fields $k$ for which all the strongly ambiguous classes of $k / \mathbb{Q}(i)$ capitulate in $k^{(*)} \subset(k / \mathbb{Q}(i))^{*}$.

Let $\mathbb{k}=\mathbb{Q}(\sqrt{d}, i)$ and $\mathbb{K}$ be an unramified quadratic extension of $\mathbb{k}$ that is abelian over $\mathbb{Q}$. Denote by $A m_{s}(\mathbb{k} / \mathbb{Q}(i))$ the group of the strongly ambiguous classes of $\mathbb{k} / \mathbb{Q}(i)$. In [6], we studied the capitulation problem in the absolutely abelian extensions of $\mathbb{k}$ for $d=2 p q$ and $p \equiv q \equiv 1(\bmod 4)$ are different primes, and in [7], we dealt with the same problem assuming $p \equiv-q \equiv 1(\bmod 4)$. In [9, 10, 11] and under the assumption

2010 Mathematics Subject Classification. 11R11, 11R16, 11R20, 11R27, 11R29.

Key words and phrases. absolute and relative genus fields, fundamental systems of units, 2-class group, capitulation, quadratic fields, biquadratic fields, multiquadratic CM-fields. 
$\mathbf{C} l_{2}(\mathbb{k}) \simeq(2,2,2)$, we studied the capitulation problem of the 2-ideal classes of $\mathbb{k}$ in its fourteen unramified extensions, within the first Hilbert 2-class field of $\mathbb{k}$, and we gave the abelian type invariants of the 2-class groups of these fourteen fields. Additionally we determined the structure of the metabelian Galois group $G=\operatorname{Gal}\left(\mathbb{k}_{2}^{(2)} / \mathbb{k}\right)$ of the second Hilbert 2-class field $\mathrm{k}_{2}^{(2)}$ of $\mathrm{k}$.

Let $q_{1} \equiv q_{2} \equiv-p \equiv-1(\bmod 4)$ be different primes and $d=p q_{1} q_{2}$. It is the purpose of the present article to pursue this research project. We will compute the capitulation kernel of $\mathbb{K} / \mathbb{k}$ and we will deduce that $\mathrm{A} m_{s}(\mathbb{k} / \mathbb{Q}(i)) \subseteq \operatorname{ker} J_{\mathbb{k}^{(*)}}$. As an application we will determine these kernels when $\mathbf{C} l_{2}(\mathrm{k})$ is of type $(2,2,2)$.

Let $k$ be a number field, during this paper, we adopt the following notations:

- $\kappa_{K}$ : the capitulation kernel of an unramified extension $K / k$.

- $\mathcal{O}_{k}$ : the ring of integers of $k$.

- $E_{k}$ : the unit group of $\mathcal{O}_{k}$.

- $W_{k}$ : the group of roots of unity contained in $k$.

- $k^{+}$: the maximal real subfield of $k$, if it is a CM-field.

- $Q_{k}=\left[E_{k}: W_{k} E_{k^{+}}\right]$is Hasse's unit index, if $k$ is a CM-field.

- $q(k / \mathbb{Q})=\left[E_{k}: \prod_{i=1}^{s} E_{k_{i}}\right]$ is the unit index of $k$, if $k$ is multiquadratic, where $k_{1}, \ldots, k_{s}$ are the quadratic subfields of $k$.

- $k^{(*)}$ : the absolute genus field of $k$.

- $\mathrm{Cl}_{2}(k)$ : the 2-class group of $k$.

- $i=\sqrt{-1}$.

- $\epsilon_{m}$ : the fundamental unit of $\mathbb{Q}(\sqrt{m})$, if $m>1$ is a square-free integer, that is a generator (modulo the roots of unity) for the unit group of the ring of integers of $\mathbb{Q}(\sqrt{m})$.

- $N(a)$ : denotes the absolute norm of a number $a$, i.e., $N_{k / \mathbb{Q}}(a)$ with $a \in k$.

- $x \pm y$ means $x+y$ or $x-y$ for some numbers $x$ and $y$.

\section{Preliminary results}

Let us first collect some results that will be useful in what follows.

Let $k_{j}, 1 \leq j \leq 3$, be the three real quadratic subfields of a biquadratic real number field $K_{0}$ and $\epsilon_{j}>1$ be the fundamental unit of $k_{j}$. Since

$$
\alpha^{2} N_{K_{0} / \mathbf{Q}}(\alpha)=\prod_{j=1}^{3} N_{K_{0} / k_{j}}(\alpha)
$$

for any $\alpha \in K_{0}$, the square of any unit of $K_{0}$ is in the group generated by the $\epsilon_{j}$ 's, $1 \leq j \leq 3$. Hence, to determine a fundamental system of units of $K_{0}$ it suffices to determine which of the units in $B:=\left\{\epsilon_{1}, \epsilon_{2}, \epsilon_{3}, \epsilon_{1} \epsilon_{2}, \epsilon_{1} \epsilon_{3}, \epsilon_{2} \epsilon_{3}, \epsilon_{1} \epsilon_{2} \epsilon_{3}\right\}$ are squares in $K_{0}$ (for details see [18] or [20]).

Lemma 2.1 ([18]). A fundamental system of units of $K_{0}$ consists of three positive units chosen among

$$
B^{\prime}:=B \cup\left\{\sqrt{\eta} \mid \eta \in B \text { and } \sqrt{\eta} \in K_{0}\right\} .
$$

Lemma 2.2 ([20]). The units $\epsilon \in B$ that can be squares in $K_{0}$ are as follows:

1. $\epsilon=\epsilon_{j}$ and $N\left(\epsilon_{j}\right)=1$ with $1 \leq j \leq 3$, 
2. $\epsilon=\epsilon_{j} \epsilon_{l}$ and $N\left(\epsilon_{j}\right)=N\left(\epsilon_{l}\right)=1$ with $1 \leq j \neq l \leq 3$,

3. $\epsilon=\epsilon_{1} \epsilon_{2} \epsilon_{3}$ and $N\left(\epsilon_{1}\right)=N\left(\epsilon_{2}\right)=N\left(\epsilon_{3}\right)$.

Put $K=K_{0}(i)$, then to determine a fundamental system of units of $K$, we will use the following result that the second author has deduced from a theorem of Hasse [17, $\S 21$, Satz 15$]$.

Lemma 2.3. 2, p.18]. Let $n \geq 2$ be an integer and $\xi_{n}$ a $2^{n}$-th primitive root of unity, then

$$
\xi_{n}=\frac{1}{2}\left(\mu_{n}+\lambda_{n} i\right), \quad \text { where } \begin{aligned}
& \mu_{n}=\sqrt{2+\mu_{n-1}}, \quad \begin{array}{r}
\lambda_{n}=\sqrt{2-\mu_{n-1}}, \\
\mu_{2}=0, \lambda_{2}=2
\end{array} \quad \text { and } \quad \mu_{3}=\lambda_{3}=\sqrt{2} .
\end{aligned}
$$

Let $n_{0}$ be the greatest integer such that $\xi_{n_{0}}$ is contained in $K,\left\{\epsilon_{1}^{\prime}, \epsilon_{2}^{\prime}, \epsilon_{3}^{\prime}\right\}$ a fundamental system of units of $K_{0}$ and $\epsilon$ a unit of $K_{0}$ such that $\left(2+\mu_{n_{0}}\right) \epsilon$ is a square in $K_{0}$ (if it exists). Then a fundamental system of units of $K$ is one of the following systems:

1. $\left\{\epsilon_{1}^{\prime}, \epsilon_{2}^{\prime}, \epsilon_{3}^{\prime}\right\}$ if $\epsilon$ does not exist,

2. $\left\{\epsilon_{1}^{\prime}, \epsilon_{2}^{\prime}, \sqrt{\xi_{n_{0}} \epsilon}\right\}$ if $\epsilon$ exists; in this case $\epsilon=\epsilon_{1}^{\prime i_{1}} \epsilon_{2}^{\prime i_{2}} \epsilon_{3}^{\prime}$, where $i_{1}, i_{2} \in\{0,1\}$ (up to a permutation).

Lemma 2.4 ([1], Lemma 5). Let $d>1$ be a square-free integer and $\epsilon_{d}=x+y \sqrt{d}$, where $x, y$ are integers or semi-integers. If $N\left(\epsilon_{d}\right)=1$, then $2(x+1), 2(x-1), 2 d(x+1)$ and $2 d(x-1)$ are not squares in $\mathbb{Q}$.

Lemma $2.5\left([1\right.$, Lemma 6$)$. Let $q \equiv-1(\bmod 4)$ be a prime and $\epsilon_{q}=x+y \sqrt{q}$ be the fundamental unit of $\mathbb{Q}(\sqrt{q})$. Then $x$ is an even integer, $x \pm 1$ is a square in $\mathbb{N}$ and $2 \epsilon_{q}$ is a square in $\mathbb{Q}(\sqrt{q})$.

Lemma 2.6 ([2], 3.(1) p.19). Let $d>2$ be a square-free integer and $k=\mathbb{Q}(\sqrt{d}, i)$, put $\epsilon_{d}=x+y \sqrt{d}$.

1. If $N\left(\epsilon_{d}\right)=-1$, then $\left\{\epsilon_{d}\right\}$ is a fundamental system of units of $k$.

2. If $N\left(\epsilon_{d}\right)=1$, then $\left\{\sqrt{i \epsilon_{d}}\right\}$ is a fundamental system of units of $k$ if and only if $x \pm 1$ is a square in $\mathbb{N}$ i.e. $2 \epsilon_{d}$ is a square in $\mathbb{Q}(\sqrt{d})$. Else $\left\{\epsilon_{d}\right\}$ is a fundamental system of units of $k$.

This result is also in [21.

Lemma $2.7([5])$. Let $d \equiv 1(\bmod 4)$ be a positive square free integer and $\epsilon_{d}=x+y \sqrt{d}$ be the fundamental unit of $\mathbb{Q}(\sqrt{d})$. Assume $N\left(\epsilon_{d}\right)=1$, then

1. $x+1$ and $x-1$ are not squares in $\mathbb{N}$ i.e. $2 \epsilon_{d}$ is not a square in $\mathbb{Q}(\sqrt{d})$.

2. For all prime $p$ dividing $d, p(x+1)$ and $p(x-1)$ are not squares in $\mathbb{N}$.

\section{Fundamental system of units of some CM-fields}

As $\mathbb{k}=\mathbb{Q}\left(\sqrt{p q_{1} q_{2}}, i\right)$, so $\mathbb{k}$ admits three unramified quadratic extensions that are abelian over $\mathbb{Q}$, which are $\mathbb{K}_{1}=\mathbb{k}(\sqrt{p})=\mathbb{Q}\left(\sqrt{p}, \sqrt{q_{1} q_{2}}, i\right), \mathbb{K}_{2}=\mathbb{k}\left(\sqrt{q_{1}}\right)=\mathbb{Q}\left(\sqrt{q_{1}}, \sqrt{q_{2} p}, i\right)$ and $\mathbb{K}_{3}=\mathbb{k}\left(\sqrt{q_{2}}\right)=\mathbb{Q}\left(\sqrt{q_{2}}, \sqrt{q_{1} p}, i\right)$. Put $\epsilon_{p q_{1} q_{2}}=x+y \sqrt{p q_{1} q_{2}}$. In what follows, we determine the fundamental system of units of $\mathbb{K}_{j}, 1 \leq j \leq 3$. 
3.1. Fundamental system of units of the field $\mathbb{K}_{1}$.

We begin by determining the systems of fundamental units of $\mathbb{K}_{1}^{+}$and $\mathbb{K}_{1}$.

Proposition 3.1. Keep the previous notations. Then $Q_{\mathbb{K}_{1}}=1$ and

1. If $2 p(x \pm 1)$ is a square in $\mathbb{N}$, then $\left\{\epsilon_{p}, \epsilon_{q_{1} q_{2}}, \sqrt{\epsilon_{p q_{1} q_{2}}}\right\}$ is a fundamental system of units of both of $\mathbb{K}_{1}^{+}$and $\mathbb{K}_{1}$.

2. Else $\left\{\epsilon_{p}, \epsilon_{q_{1} q_{2}}, \sqrt{\epsilon_{q_{1} q_{2}} \epsilon_{p q_{1} q_{2}}}\right\}$ is a fundamental system of units of both of $\mathbb{K}_{1}^{+}$and $\mathbb{K}_{1}$.

Proof. As $N\left(\epsilon_{p}\right)=-1$, then by Lemma 2.2 only $\epsilon_{q_{1} q_{2}}, \epsilon_{p q_{1} q_{2}}$ and $\epsilon_{q_{1} q_{2}} \epsilon_{p q_{1} q_{2}}$ can be squares in $\mathbb{K}_{1}^{+}$.

Put $\epsilon_{q_{1} q_{2}}=a+b \sqrt{q_{1} q_{2}}$, then $a^{2}-1=b^{2} q_{1} q_{2}$. Hence by Lemmas 2.4 and 2.7 we get that only the number $2 q_{1}(a \pm 1)$ (i.e. $2 q_{2}(a \pm 1)$ ) is a square in $\mathbb{N}$. So there exist $b_{1}$ and $b_{2}$ in $\mathbb{Z}$ such that

$$
\left\{\begin{array}{l}
a \pm 1=2 b_{1}^{2} q_{1} \\
a \mp 1=2 b_{2}^{2} q_{2}
\end{array}\right.
$$

therefore $\sqrt{\epsilon_{q_{1} q_{2}}}=b_{1} \sqrt{q_{1}}+b_{2} \sqrt{q_{2}}$, which implies that $q_{1} \epsilon_{q_{1} q_{2}}$ and $q_{2} \epsilon_{q_{1} q_{2}}$ are squares in $\mathbb{K}_{1}^{+}$but $\epsilon_{q_{1} q_{2}}$ is not.

Since $N\left(\epsilon_{p q_{1} q_{2}}\right)=1$, then $x^{2}-1=y^{2} p q_{1} q_{2}$. Hence Lemmas 2.4 and 2.7 allowed us to distinguish the following cases:

a. If $2 p(x \pm 1)$ is a square in $\mathbb{N}$, then $\sqrt{\epsilon_{p q_{1} q_{2}}}=y_{1} \sqrt{p}+y_{2} \sqrt{q_{1} q_{2}}$, hence $\epsilon_{p q_{1} q_{2}}$ is a square in $\mathbb{K}_{1}^{+}$.

b. If $2 q_{1}(x \pm 1)$ is a square in $\mathbb{N}$, then $\sqrt{\epsilon_{p q_{1} q_{2}}}=y_{1} \sqrt{q_{1}}+y_{2} \sqrt{p q_{2}}$, hence $q_{1} \epsilon_{p q_{1} q_{2}}$ and $p q_{2} \epsilon_{p q_{1} q_{2}}$ are squares in $\mathbb{K}_{1}^{+}$but $\epsilon_{p q_{1} q_{2}}$ is not.

c. If $2 q_{2}(x \pm 1)$ is a square in $\mathbb{N}$, then $\sqrt{\epsilon_{p q_{1} q_{2}}}=y_{1} \sqrt{q_{2}}+y_{2} \sqrt{p q_{1}}$, hence $q_{2} \epsilon_{p q_{1} q_{2}}$ and $p q_{1} \epsilon_{p q_{1} q_{2}}$ are squares in $\mathbb{K}_{1}^{+}$but $\epsilon_{p q_{1} q_{2}}$ is not.

Consequently, we have

1. If $2 p(x \pm 1)$ is a square in $\mathbb{N}$, then $\epsilon_{p q_{1} q_{2}}$ is a square in $\mathbb{K}_{1}^{+}$. Thus Lemmas 2.1 and 2.3 yield that $\left\{\epsilon_{p}, \epsilon_{q_{1} q_{2}}, \sqrt{\epsilon_{p q_{1} q_{2}}}\right\}$ is a fundamental system of units of both of $\mathbb{K}_{1}^{+}$and $\mathbb{K}_{1}$.

2. If $2 q_{1}(x \pm 1)$ or $2 q_{2}(x \pm 1)$ is a square in $\mathbb{N}$, then $q_{1} \epsilon_{p q_{1} q_{2}}$ or $q_{2} \epsilon_{p q_{1} q_{2}}$ is a square in $\mathbb{K}_{1}^{+}$. As $q_{1} \epsilon_{q_{1} q_{2}}$ and $q_{2} \epsilon_{q_{1} q_{2}}$ are squares in $\mathbb{K}_{1}^{+}$, so $\epsilon_{q_{1} q_{2}} \epsilon_{p q_{1} q_{2}}$ is a square in $\mathbb{K}_{1}^{+}$. Thus Lemmas 2.1 and 2.3 yield that $\left\{\epsilon_{p}, \epsilon_{q_{1} q_{2}}, \sqrt{\epsilon_{q_{1} q_{2}} \epsilon_{p q_{1} q_{2}}}\right\}$ is a fundamental system of units of both of $\mathbb{K}_{1}^{+}$and $\mathbb{K}_{1}$.

\subsection{Fundamental system of units of the field $\mathbb{K}_{2}$.}

Let us now determine the fundamental system of units's of $\mathbb{K}_{2}^{+}=\mathbb{Q}\left(\sqrt{q_{1}}, \sqrt{p q_{2}}\right)$ and $\mathbb{K}_{2}=\mathbb{Q}\left(\sqrt{q_{1}}, \sqrt{p q_{2}}, i\right)$.

Proposition 3.2. Keep the previous notations and put $\epsilon_{p q_{2}}=a+b \sqrt{p q_{2}}$. Then $Q_{\mathbb{K}_{2}}=2$. Moreover we have:

1. Assume $2 q_{1}(x \pm 1)$ is a square in $\mathbb{N}$, then

i. If $a \pm 1$ is a square in $\mathbb{N}$, then $\left\{\epsilon_{q_{1}}, \sqrt{\epsilon_{q_{1}} \epsilon_{p q_{2}}}, \sqrt{\epsilon_{p q_{1} q_{2}}}\right\}$ is a fundamental system of units of $\mathbb{K}_{2}^{+}$, and that of $\mathbb{K}_{2}$ is $\left\{\sqrt{\epsilon_{q_{1}} \epsilon_{p q_{2}}}, \sqrt{\epsilon_{p q_{1} q_{2}}}, \sqrt{i \epsilon_{q_{1}}}\right\}$. 
ii. Else $\left\{\epsilon_{q_{1}}, \epsilon_{p q_{2}}, \sqrt{\epsilon_{p q_{1} q_{2}}}\right\}$ is a fundamental system of units of $\mathbb{K}_{2}^{+}$, and that of $\mathbb{K}_{2}$ is $\left\{\epsilon_{p q_{2}}, \sqrt{\epsilon_{p q_{1} q_{2}}}, \sqrt{i \epsilon_{q_{1}}}\right\}$.

2. Assume $2 q_{1}(x \pm 1)$ is not a square in $\mathbb{N}$, then

i. If $a \pm 1$ is a square in $\mathbb{N}$, then $\left\{\epsilon_{q_{1}}, \sqrt{\epsilon_{q_{1}} \epsilon_{p q_{2}}}, \epsilon_{p q_{1} q_{2}}\right\}$ is a fundamental system of units of $\mathbb{K}_{2}^{+}$, and that of $\mathbb{K}_{2}$ is $\left\{\sqrt{\epsilon_{q_{1}} \epsilon_{p q_{2}}}, \epsilon_{p q_{1} q_{2}}, \sqrt{i \epsilon_{q_{1}}}\right\}$.

ii. If $p(a \pm 1)$ is a square in $\mathbb{N}$, then $\left\{\epsilon_{q_{1}}, \epsilon_{p q_{2}}, \sqrt{\epsilon_{q_{1}} \epsilon_{p q_{2}} \epsilon_{p q_{1} q_{2}}}\right\}$ is a fundamental system of units of $\mathbb{K}_{2}^{+}$, and that of $\mathbb{K}_{2}$ is $\left\{\epsilon_{p q_{2}}, \sqrt{\epsilon_{q_{1}} \epsilon_{p q_{2}} \epsilon_{p q_{1} q_{2}}}, \sqrt{i \epsilon_{q_{1}}}\right\}$.

iii. If $2 p(a \pm 1)$ is a square in $\mathbb{N}$, then $\left\{\epsilon_{q_{1}}, \epsilon_{p q_{2}}, \sqrt{\epsilon_{p q_{2}} \epsilon_{p q_{1} q_{2}}}\right\}$ is a fundamental system of units of $\mathbb{K}_{2}^{+}$, and that of $\mathbb{K}_{2}$ is $\left\{\epsilon_{p q_{2}}, \sqrt{\epsilon_{p q_{2}} \epsilon_{p q_{1} q_{2}}}, \sqrt{i \epsilon_{q_{1}}}\right\}$.

Proof. By Lemma 2.2 the units that can be squares in $\mathbb{K}_{2}$ are: $\epsilon_{q_{1}}, \epsilon_{p q_{2}}, \epsilon_{p q_{1} q_{2}}, \epsilon_{q_{1}} \epsilon_{p q_{2}}$, $\epsilon_{q_{1}} \epsilon_{p q_{1} q_{2}}, \epsilon_{p q_{1}} \epsilon_{p q_{1} q_{2}}$ and $\epsilon_{q_{1}} \epsilon_{p q_{2}} \epsilon_{p q_{1} q_{2}}$.

According to Lemma 2.5, $2 \epsilon_{q_{1}}$ is a square in $\mathbb{K}_{2}^{+}$but $\epsilon_{q_{1}}$ is not.

Put $\epsilon_{p q_{2}}=a+b \sqrt{p q_{2}}$, then $a^{2}-1=b^{2} p q_{2}$. Hence Lemma 2.4 allowed us to distinguish the following cases:

a. If $a \pm 1$ is a square in $\mathbb{N}$, then there exist $b_{1}$ and $b_{2}$ in $\mathbb{Z}$ such that

$$
\left\{\begin{array}{l}
a \pm 1=b_{1}^{2} \\
a \mp 1=b_{2}^{2} p q_{2}
\end{array}\right.
$$

thus $\sqrt{2 \epsilon_{p q_{2}}}=b_{1}+b_{2} \sqrt{p q_{2}}$. Therefore $2 \epsilon_{p q_{2}}$ a square in $\mathbb{K}_{1}^{+}$but $\epsilon_{p q_{2}}$ is not.

b. If $p(a \pm 1)$ is a square in $\mathbb{N}$, then there exist $b_{1}$ and $b_{2}$ in $\mathbb{Z}$ such that

$$
\left\{\begin{array}{l}
a \pm 1=b_{1}^{2} p \\
a \mp 1=b_{2}^{2} q_{2}
\end{array}\right.
$$

thus $\sqrt{2 \epsilon_{p q_{2}}}=b_{1} \sqrt{p}+b_{2} \sqrt{q_{2}}$. Therefore $2 p \epsilon_{p q_{2}}$ and $2 q_{2} \epsilon_{q_{1} q_{2}}$ are squares in $\mathbb{K}_{2}^{+}$but $\epsilon_{p q_{2}}$ and $2 \epsilon_{p q_{2}}$ are not.

c. If $2 p(a \pm 1)$ is a square in $\mathbb{N}$, then there exist $b_{1}$ and $b_{2}$ in $\mathbb{Z}$ such

$$
\left\{\begin{array}{l}
a \pm 1=2 b_{1}^{2} p \\
a \mp 1=2 b_{2}^{2} q_{2}
\end{array}\right.
$$

thus $\sqrt{\epsilon_{p q_{2}}}=b_{1} \sqrt{p}+b_{2} \sqrt{q_{2}}$. Therefore $p \epsilon_{p q_{2}}$ and $q_{2} \epsilon_{p q_{2}}$ are squares in $\mathbb{K}_{2}^{+}$but $\epsilon_{p q_{2}}$ is not.

As $N\left(\epsilon_{p q_{1} q_{2}}\right)=1$, then $x^{2}-1=y^{2} p q_{1} q_{2}$; hence Lemmas 2.4 and 2.7 allowed us to distinguish the following cases:

a'. If $2 p(x \pm 1)$ is a square in $\mathbb{N}$, then $\sqrt{\epsilon_{p q_{1} q_{2}}}=y_{1} \sqrt{p}+y_{2} \sqrt{q_{1} q_{2}}$, thus $p \epsilon_{p q_{1} q_{2}}$ and $q_{1} q_{2} \epsilon_{p q_{1} q_{2}}$ are squares in $\mathbb{K}_{2}^{+}$but $\epsilon_{p q_{1} q_{2}}$ is not.

b'. If $2 q_{1}(x \pm 1)$ is a square in $\mathbb{N}$, then $\sqrt{\epsilon_{p q_{1} q_{2}}}=y_{1} \sqrt{q_{1}}+y_{2} \sqrt{p q_{2}}$, thus $\epsilon_{p q_{1} q_{2}}$ is a square in $\mathbb{K}_{2}^{+}$.

c'. If $2 q_{2}(x \pm 1)$ is a square in $\mathbb{N}$, then $\sqrt{\epsilon_{p q_{1} q_{2}}}=y_{1} \sqrt{q_{2}}+y_{2} \sqrt{p q_{1}}$, thus $q_{2} \epsilon_{p q_{1} q_{2}}$ and $p q_{1} \epsilon_{p q_{1} q_{2}}$ are squares in $\mathbb{K}_{1}^{+}$but $\epsilon_{p q_{1} q_{2}}$ is not.

Consequently, we have

1. Assume $2 q_{1}(x \pm 1)$ is a square in $\mathbb{N}$, then $\epsilon_{p q_{1} q_{2}}$ is a square in $\mathbb{K}_{2}^{+}$.

i. If $a \pm 1$ is a square in $\mathbb{N}$, then $2 \epsilon_{p q_{2}}$ is a square in $\mathbb{K}_{2}^{+}$; thus $\epsilon_{q_{1}} \epsilon_{p q_{2}}$ is a square in $\mathbb{K}_{2}^{+}$, since $2 \epsilon_{q_{1}}$ is. Therefore, by Lemma $2.1\left\{\epsilon_{q_{1}}, \sqrt{\epsilon_{q_{1}} \epsilon_{p q_{2}}}, \sqrt{\epsilon_{p q_{1} q_{2}}}\right\}$ is a fundamental 
system of units of $\mathbb{K}_{2}^{+}$, and according to Lemma $2.3\left\{\sqrt{\epsilon_{q_{1}} \epsilon_{p q_{2}}}, \sqrt{\epsilon_{p q_{1} q_{2}}}, \sqrt{i \epsilon_{q_{1}}}\right\}$ is a fundamental system of units of $\mathbb{K}_{2}$.

ii. Else $\epsilon_{p q_{1} q_{2}}$ will be a square in $\mathbb{K}_{2}^{+}$; hence by Lemma $2.1\left\{\epsilon_{q_{1}}, \epsilon_{p q_{2}}, \sqrt{\epsilon_{p q_{1} q_{2}}}\right\}$ is a fundamental system of units of $\mathbb{K}_{2}^{+}$, and according to Lemma $2.3\left\{\epsilon_{p q_{2}}, \sqrt{\epsilon_{p q_{1} q_{2}}}, \sqrt{i \epsilon_{q_{1}}}\right\}$ is a fundamental system of units of $\mathbb{K}_{2}$.

2. Assume $2 q_{1}(x \pm 1)$ is not a square in $\mathbb{N}$, then $\epsilon_{p q_{1} q_{2}}$ is not a square in $\mathbb{K}_{2}^{+}$.

i. If $a \pm 1$ is a square in $\mathbb{N}$, then $2 \epsilon_{p q_{2}}$ is a square in $\mathbb{K}_{2}^{+}$; hence $\epsilon_{q_{1}} \epsilon_{p q_{2}}$ is a square in $\mathbb{K}_{2}^{+}$, since $2 \epsilon_{q_{1}}$ is a square in $\mathbb{N}$. Thus by Lemma 2.1 $\left\{\epsilon_{q_{1}}, \sqrt{\epsilon_{q_{1}} \epsilon_{p q_{2}}}, \epsilon_{p q_{1} q_{2}}\right\}$ is a fundamental system of units of $\mathbb{K}_{2}^{+}$, and according to Lemma $2.3\left\{\sqrt{\epsilon_{q_{1}} \epsilon_{p q_{2}}}, \epsilon_{p q_{1} q_{2}}, \sqrt{i \epsilon_{q_{1}}}\right\}$ is a fundamental system of units of $\mathbb{K}_{2}$.

ii. If $p(a \pm 1)$ is a square in $\mathbb{N}$, then $2 p \epsilon_{p q_{2}}$ and $2 q_{2} \epsilon_{p q_{2}}$ are squares in $\mathbb{K}_{2}^{+}$. On the other hand, we have $p \epsilon_{p q_{1} q_{2}}$ or $q_{2} \epsilon_{p q_{1} q_{2}}$ is a square in $\mathbb{K}_{1}^{+}$, thus $\epsilon_{q_{1}} \epsilon_{p q_{2}} \epsilon_{p q_{1} q_{2}}$ is a square in $\mathbb{K}_{2}^{+}$, since $2 \epsilon_{q_{1}}$ is a square in $\mathbb{N}$. Therefore by Lemma $2.1\left\{\epsilon_{q_{1}}, \epsilon_{p q_{2}}, \sqrt{\epsilon_{q_{1}} \epsilon_{p q_{2}} \epsilon_{p q_{1} q_{2}}}\right\}$ is a fundamental system of units of $\mathbb{K}_{2}^{+}$, and according to Lemma 2.3 $\left\{\epsilon_{p q_{2}}, \sqrt{\epsilon_{q_{1}} \epsilon_{p q_{2}} \epsilon_{p q_{1} q_{2}}}, \sqrt{i \epsilon_{q_{1}}}\right\}$ is a fundamental system of units of $\mathbb{K}_{2}$.

iii. The last case is treated similarly.

3.3. Fundamental system of units of the field $\mathbb{K}_{3}$.

Since $q_{1}$ and $q_{2}$ play symmetrical roles, then the fundamental system of units's of $\mathbb{K}_{3}^{+}=$ $\mathbb{Q}\left(\sqrt{q_{2}}, \sqrt{p q_{1}}\right)$ and $\mathbb{K}_{3}=\mathbb{Q}\left(\sqrt{q_{2}}, \sqrt{p q_{1}}, i\right)$ are easily deduced.

Proposition 3.3. Keep the previous notations and put $\epsilon_{p q_{1}}=a+b \sqrt{p q_{1}}$. Then $Q_{\mathbb{K}_{3}}=2$. Moreover we have.

1.) Assume $2 q_{2}(x \pm 1)$ is a square in $\mathbb{N}$, then

i. If $a \pm 1$ is a square in $\mathbb{N}$, then $\left\{\epsilon_{q_{2}}, \sqrt{\epsilon_{q_{2}} \epsilon_{p q_{1}}}, \sqrt{\epsilon_{p q_{1} q_{2}}}\right\}$ is a fundamental system of units of $\mathbb{K}_{3}^{+}$, and that of $\mathbb{K}_{3}$ is $\left\{\sqrt{\epsilon_{q_{2}} \epsilon_{p q_{1}}}, \sqrt{\epsilon_{p q_{1} q_{2}}}, \sqrt{i \epsilon_{q_{2}}}\right\}$.

ii. Else $\left\{\epsilon_{q_{2}}, \epsilon_{p q_{1}}, \sqrt{\epsilon_{p q_{1} q_{2}}}\right\}$ is a fundamental system of units of $\mathbb{K}_{3}^{+}$, and that of $\mathbb{K}_{3}$ is $\left\{\epsilon_{p q_{1}}, \sqrt{\epsilon_{p q_{1} q_{2}}}, \sqrt{i \epsilon_{q_{2}}}\right\}$.

2.) Assume $2 q_{2}(x \pm 1)$ is not a square in $\mathbb{N}$, then

i. If $a \pm 1$ is a square in $\mathbb{N}$, then $\left\{\epsilon_{q_{2}}, \sqrt{\epsilon_{q_{2}} \epsilon_{p q_{1}}}, \epsilon_{p q_{1} q_{2}}\right\}$ is a fundamental system of units of $\mathbb{K}_{3}^{+}$, and that of $\mathbb{K}_{3}$ is $\left\{\sqrt{\epsilon_{q_{2}} \epsilon_{p q_{1}}}, \epsilon_{p q_{1} q_{2}}, \sqrt{i \epsilon_{q_{2}}}\right\}$.

ii. If $p(a \pm 1)$ is a square in $\mathbb{N}$, then $\left\{\epsilon_{q_{2}}, \epsilon_{p q_{1}}, \sqrt{\epsilon_{q_{2}} \epsilon_{p q_{1}} \epsilon_{p q_{1} q_{2}}}\right\}$ is a fundamental system of units of $\mathbb{K}_{3}^{+}$, and that of $\mathbb{K}_{2}$ is $\left\{\epsilon_{p q_{1}}, \sqrt{\epsilon_{q_{2}} \epsilon_{p q_{1}} \epsilon_{p q_{1} q_{2}}}, \sqrt{i \epsilon_{q_{2}}}\right\}$.

iii. If $2 p(a \pm 1)$ is a square in $\mathbb{N}$, then $\left\{\epsilon_{q_{2}}, \epsilon_{p q_{1}}, \sqrt{\epsilon_{p q_{1}} \epsilon_{p q_{1} q_{2}}}\right\}$ is a fundamental system of units of $\mathbb{K}_{3}^{+}$, and that of $\mathbb{K}_{3}$ is $\left\{\epsilon_{p q_{1}}, \sqrt{\epsilon_{p q_{1}} \epsilon_{p q_{1} q_{2}}}, \sqrt{i \epsilon_{q_{2}}}\right\}$.

\section{The ambiguous classes of $\mathbb{k} / \mathbb{Q}(i)$}

Let $F=\mathbb{Q}(i)$ and $\mathbb{k}=\mathbb{Q}\left(\sqrt{p q_{1} q_{2}}, i\right)$. We denote by $\mathrm{A} m(\mathbb{k} / F)$ the group of the ambiguous classes of $\mathbb{k} / F$ and by $\mathrm{A}_{s}(\mathbb{k} / F)$ the subgroup of $\mathrm{A} m(\mathbb{k} / F)$ generated by the strongly ambiguous classes. As $p \equiv 1(\bmod 4)$, so there exist $e$ and $f$ in $\mathbb{N}$ such that $p=e^{2}+4 f^{2}=\pi_{1} \pi_{2}$. Put $\pi_{1}=e+2 i f$ and $\pi_{2}=e-2 i f$. Let $\mathcal{H}_{j}$ (resp. $\mathcal{Q}_{j}$ ) be the prime ideal of $\mathbb{k}$ above $\pi_{j}$ (resp. $q_{j}$ ), where $j \in\{1,2\}$. It is easy to see that $\mathcal{H}_{j}^{2}=\left(\pi_{j}\right)$ and $\mathcal{Q}_{j}^{2}=\left(q_{j}\right)$. Therefore $\left[\mathcal{Q}_{j}\right]$ and $\left[\mathcal{H}_{j}\right]$ are in $\mathrm{A} m_{s}(\mathbb{k} / F)$, for all $j \in\{1,2\}$. 
Keep the notation $\epsilon_{p q_{1} q_{2}}=x+y \sqrt{p q_{1} q_{2}}$. In this section, we will determine generators of $\mathrm{A} m_{s}(\mathrm{k} / F)$ and $\mathrm{A} m(\mathrm{k} / F)$. Let us first prove the following result.

Lemma 4.1. Consider the prime ideals $\mathcal{H}_{j}$ and $\mathcal{Q}_{j}$ of $\mathrm{k}, 1 \leq j \leq 2$.

1. If $2 p(x \pm 1)$ is a square in $\mathbb{N}$, then $\left|\left\langle\left[\mathcal{H}_{1}\right],\left[\mathcal{Q}_{1}\right]\right\rangle\right|=4$.

2. Else $\left|\left\langle\left[\mathcal{H}_{1}\right],\left[\mathcal{H}_{2}\right]\right\rangle\right|=4$

Proof. Since $\mathcal{H}_{j}^{2}=\left(\pi_{j}\right), 1 \leq j \leq 2$, and since also $\sqrt{e^{2}+(2 f)^{2}}=\sqrt{p} \notin \mathbb{Q}\left(\sqrt{p q_{1} q_{2}}\right)$, so, according to [4, Proposition 1], $\mathcal{H}_{j}$ are not principal in $\mathrm{k}$.

1. If $2 p(x \pm 1)$ is a square in $\mathbb{N}$, and since $\left(\mathcal{H}_{1} \mathcal{H}_{2}\right)^{2}=\left(p_{1}\right), \mathcal{Q}_{j}^{2}=\left(q_{j}\right)$ and $\left(\mathcal{H}_{1} \mathcal{Q}_{j}\right)^{2}=$ $\left(q_{j}\right)$, hence by [4, Proposition 2 and Remark 1], $\mathcal{H}_{1} \mathcal{H}_{2}$ is principal in $\mathbb{k}$ and $\mathcal{Q}_{j}, \mathcal{H}_{1} \mathcal{Q}_{j}$ are not. Thus the result.

2. If $2 p(x \pm 1)$ is not a square in $\mathbb{N}$, i.e. $2 q_{1}(x \pm 1)$ or $2 q_{2}(x \pm 1)$ is a square in $\mathbb{N}$; then $\mathcal{H}_{1} \mathcal{H}_{2}$ is not principal in $\mathrm{k}$ and $\mathcal{Q}_{1}$ or $\mathcal{Q}_{2}$ is (by [4, Proposition 2]). On the other hand, if $\mathcal{Q}_{1}$ (resp. $\mathcal{Q}_{2}$ ) is principal, then $\left[\mathcal{H}_{1} \mathcal{H}_{2}\right]=\left[\mathcal{Q}_{2}\right]\left(\right.$ resp. $\left.\left[\mathcal{H}_{1} \mathcal{H}_{2}\right]=\left[\mathcal{Q}_{1}\right]\right)$.

Determine now generators of $\mathrm{A} m_{s}(\mathbb{k} / F)$ and $\mathrm{A} m(\mathbb{k} / F)$. According to the ambiguous class number formula $([12])$, the genus number, $\left[(\mathbb{k} / F)^{*}: \mathbb{k}\right]$, is given by:

$$
|\mathrm{A} m(\mathbb{k} / F)|=\left[(\mathbb{k} / F)^{*}: \mathbb{k}\right]=\frac{h(F) 2^{t-1}}{\left[E_{F}: E_{F} \cap N_{\mathbb{k} / F}\left(\mathbb{k}^{\times}\right)\right]},
$$

where $h(F)$ is the class number of $F$ and $t$ is the number of finite and infinite primes of $F$ ramified in $\mathrm{k} / F$. Moreover as the class number of $F$ is equal to 1 , so the formula (1) yields that

$$
|\mathrm{A} m(\mathbb{k} / F)|=\left[(\mathbb{k} / F)^{*}: \mathbb{k}\right]=2^{r},
$$

where $r=\operatorname{rank} l_{2}(\mathbb{k})=t-e-1$ and $2^{e}=\left[E_{F}: E_{F} \cap N_{\mathrm{k} / F}\left(\mathbb{k}^{\times}\right)\right]$(see for example [22]). The relation between $|\mathrm{A} m(\mathbb{k} / F)|$ and $\left|\mathrm{A} m_{s}(\mathbb{k} / F)\right|$ is given by the following formula (see for example [13]):

$$
\frac{|\mathrm{A} m(\mathbb{k} / F)|}{\left|\mathrm{A} m_{s}(\mathbb{k} / F)\right|}=\left[E_{F} \cap N_{\mathbb{k} / F}\left(\mathbb{k}^{\times}\right): N_{\mathbb{k} / F}\left(E_{\mathbb{k}}\right)\right] .
$$

To continue, we need the following lemma.

Lemma 4.2. Let $p \equiv-q_{1} \equiv-q_{2} \equiv 1(\bmod 4)$ be different primes, $F=\mathbb{Q}(i)$ and $\mathrm{k}=\mathbb{Q}\left(\sqrt{p q_{1} q_{2}}, i\right)$.

1. If $p \equiv 1(\bmod 8)$, then $i$ is a norm in $\mathrm{k} / F$.

2. If $p \equiv 5(\bmod 8)$, then $i$ is not a norm in $\mathrm{k} / F$.

Proof. We proceed as in Lemma 11 of [7].

Proposition 4.3. Let $(\mathrm{k} / F)^{*}$ denote the relative genus field of $\mathrm{k} / F$. Then

1. i. If $p \equiv 1(\bmod 8)$, then $\mathbb{k}^{(*)} \varsubsetneqq(\mathbb{k} / F)^{*}$ and $\left[(\mathbb{k} / F)^{*}: \mathbb{k}^{(*)}\right]=2$.

ii. Else $\mathbb{k}^{(*)}=(\mathrm{k} / F)^{*}$.

2. Assume $p \equiv 1(\bmod 8)$.

i. If $2 p(x \pm 1)$ is a square in $\mathbb{N}$, then $\operatorname{Am}_{s}(\mathbb{k} / \mathbb{Q}(i))=\left\langle\left[\mathcal{H}_{1}\right],\left[\mathcal{Q}_{1}\right]\right\rangle$.

ii. Else, $\operatorname{Am}_{s}(\mathbb{k} / \mathbb{Q}(i))=\left\langle\left[\mathcal{H}_{1}\right],\left[\mathcal{H}_{2}\right]\right\rangle$. 
iii. there exist an unambiguous ideal $\mathcal{I}$ in $\mathrm{k} / \mathbb{Q}(i)$ of order 2 such that

$$
\operatorname{Am}(\mathbb{k} / \mathbb{Q}(i))= \begin{cases}\left\langle\left[\mathcal{H}_{1}\right],\left[\mathcal{Q}_{1}\right],[\mathcal{I}]\right\rangle, & \text { if } 2 p(x \pm 1) \text { is a square in } \mathbb{N} \\ \left\langle\left[\mathcal{H}_{1}\right],\left[\mathcal{H}_{2}\right],[\mathcal{I}]\right\rangle, & \text { otherwise. }\end{cases}
$$

3. Assume $p \equiv 5(\bmod 8)$, then

$$
\operatorname{Am}(\mathbb{k} / \mathbb{Q}(i))=\operatorname{Am}_{s}(\mathbb{k} / \mathbb{Q}(i))= \begin{cases}\left\langle\left[\mathcal{H}_{1}\right],\left[\mathcal{Q}_{1}\right]\right\rangle, & \text { if } 2 p(x \pm 1) \text { is a square in } \mathbb{N} \\ \left\langle\left[\mathcal{H}_{1}\right],\left[\mathcal{H}_{2}\right]\right\rangle, & \text { otherwise. }\end{cases}
$$

Proof. 1. As $\mathbb{k}=\mathbb{Q}\left(\sqrt{p q_{1} q_{2}}, i\right)$, so $\left[\mathbb{k}^{(*)}: \mathbb{k}\right]=4$. Moreover, according to [22, Proposition 2, p. 90], $r=\operatorname{rank} \mathbf{C} l_{2}(\mathbb{k})=3$ if $p \equiv 1(\bmod 8)$ and $r=\operatorname{rank} \mathbf{C l} l_{2}(\mathbb{k})=2$ if $p \equiv 5$ $(\bmod 8)$, so $\left[(\mathbb{k} / F)^{*}: \mathbb{k}\right]=4$ or 8 . Hence $\left[(\mathbb{k} / F)^{*}: \mathbb{k}^{(*)}\right]=1$ or 2 , and the results derived.

2. Note first that, by Lemma 2.7, $x+1$ and $x-1$ are never squares in $\mathbb{N}$. Thus from Lemma 2.6 we get $E_{\mathbb{k}}=\left\langle i, \epsilon_{p q_{1} q_{2}}\right\rangle$.

Assume $p \equiv 1(\bmod 8)$, hence $i$ is a norm in $\mathrm{k} / \mathbb{Q}(i)$ (Lemma 4.2), thus Formula (3) yields that

$$
\frac{|\operatorname{Am}(\mathbb{k} / \mathbb{Q}(i))|}{\left|\operatorname{Am}_{s}(\mathbb{k} / \mathbb{Q}(i))\right|}=\left[E_{\mathbb{Q}(i)} \cap N_{\mathbb{k} / \mathbb{Q}(i)}\left(\mathbb{k}^{\times}\right): N_{\mathbb{k} / \mathbb{Q}(i)}\left(E_{\mathbb{k}}\right)\right]=2
$$

since $\left.\left[E_{\mathbb{Q}(i)} \cap N_{\mathrm{k} / \mathbb{Q}(i)}\left(\mathbb{k}^{\times}\right): N_{\mathbb{k} / \mathbb{Q}(i)}\left(E_{\mathbb{k}}\right)\right]=[\langle i\rangle:<-1\rangle\right]=2$.

On the other hand, as $p \equiv 1(\bmod 8)$, we have just shown that $r=3$. Therefore $|\operatorname{Am}(\mathbb{k} / \mathbb{Q}(i))|=2^{4}$ and thus $\left|\operatorname{Am}_{s}(\mathbb{k} / \mathbb{Q}(i))\right|=4$

i. If $2 p(x \pm 1)$ is a square in $\mathbb{N}$ which is equivalent to $2 q_{1} q_{2}(x \pm 1)$ is a square in $\mathbb{N}$, then $\operatorname{Am}(\mathbb{k} / \mathbb{Q}(i))=2 \operatorname{Am}_{s}(\mathbb{k} / \mathbb{Q}(i))$, hence by Lemma 4.1 we get

$$
\operatorname{Am}_{s}(\mathbb{k} / \mathbb{Q}(i))=\left\langle\left[\mathcal{H}_{1}\right],\left[\mathcal{Q}_{1}\right]\right\rangle .
$$

ii. If $2 q_{1}(x \pm 1)$ or $2 q_{2}(x \pm 1)$ is a square in $\mathbb{N}$, then Lemma 4.1 yields that

$$
\operatorname{Am}_{s}(\mathbb{k} / \mathbb{Q}(i))=\left\langle\left[\mathcal{H}_{1}\right],\left[\mathcal{H}_{2}\right]\right\rangle .
$$

Consequently, in the two cases there exists an unambiguous ideal $\mathcal{I}$ in $\mathrm{k} / F$ of order 2 such that

$$
\operatorname{Am}(\mathbb{k} / \mathbb{Q}(i))= \begin{cases}\left\langle\left[\mathcal{H}_{1}\right],\left[\mathcal{Q}_{1}\right],[\mathcal{I}]\right\rangle, & \text { if } 2 p(x \pm 1) \text { is a square in } \mathbb{N} \\ \left\langle\left[\mathcal{H}_{1}\right],\left[\mathcal{H}_{2}\right],[\mathcal{I}]\right\rangle, & \text { else. }\end{cases}
$$

By Chebotarev theorem, $\mathcal{I}$ can always be chosen as a prime ideal of $\mathbb{k}$ above a prime $\ell$ in $\mathbb{Q}$, which splits completely in $\mathbb{k}$.

3. Assume $p \equiv 5(\bmod 8)$, hence $i$ is not a norm in $\mathbb{k} / \mathbb{Q}(i)$ (Lemma 4.2). Proceeding similarly as in 2 , we get

$$
\operatorname{Am}(\mathbb{k} / \mathbb{Q}(i))=\operatorname{Am}_{s}(\mathbb{k} / \mathbb{Q}(i))= \begin{cases}\left\langle\left[\mathcal{H}_{1}\right],\left[\mathcal{Q}_{1}\right]\right\rangle, & \text { if } 2 p(x \pm 1) \text { is a square in } \mathbb{N}, \\ \left\langle\left[\mathcal{H}_{1}\right],\left[\mathcal{H}_{2}\right]\right\rangle, & \text { else. }\end{cases}
$$

This completes the proof. 


\section{Capitulation}

Let $p, q_{1}$ and $q_{2}$ be primes satisfying $p \equiv-q_{1} \equiv-q_{2} \equiv 1(\bmod 4)$. Set $\mathbb{k}=$ $\mathbb{Q}\left(\sqrt{p q_{1} q_{2}}, i\right)$ and denote by $\mathbb{k}^{(*)}$ the genus field of $\mathbb{k}$, then $\mathbb{k}^{(*)}=\mathbb{Q}\left(\sqrt{p}, \sqrt{q_{1}}, \sqrt{q_{2}}, i\right)$. The unramified quadratic extensions of $\mathbb{k}$, abelian over $\mathbb{Q}$, are $\mathbb{K}_{1}=\mathbb{k}(\sqrt{p})=\mathbb{Q}\left(\sqrt{p}, \sqrt{q_{1} q_{2}}, i\right)$, $\mathbb{K}_{2}=\mathbb{k}\left(\sqrt{q_{1}}\right)=\mathbb{Q}\left(\sqrt{q_{1}}, \sqrt{p q_{2}}, i\right)$ and $\mathbb{K}_{3}=\mathbb{k}_{(}\left(\sqrt{q_{2}}\right)=\mathbb{Q}\left(\sqrt{q_{2}}, \sqrt{p q_{1}}, i\right)$. Keep the notations $\epsilon_{p q_{1} q_{2}}=x+y \sqrt{p q_{1} q_{2}}$ denoting the fundamental unit of $\mathbb{Q}\left(\sqrt{p q_{1} q_{2}}\right)$ and $p=$ $e^{2}+4 f^{2}=\pi_{1} \pi_{2}$, where $\pi_{1}=e+2 i f, \pi_{2}=e-2 i f$. Let $Q_{\mathbb{k}}$ be the unit index of $\mathbb{k}$, and $\mathcal{H}_{j}$ be the ideal of $\mathrm{kk}$ lies above $\pi_{j}$. Denote also by $\mathcal{Q}_{j}$ the prime ideal of $\mathrm{k}$ above $q_{j}$, $j=1,2$.

In this section, we will determine the classes of $\mathbf{C} l_{2}(\mathbb{k})$, the 2-class group of $\mathbb{k}$, that capitulate in $\mathbb{K}_{j}$, for all $j \in\{1,2,3\}$. For this we need the following theorem.

Theorem 5.1 ([14]). Let $K / k$ be a cyclic extension of prime degree, then the number of classes that capitulate in $K / k$ is: $[K: k]\left[E_{k}: N_{K / k}\left(E_{K}\right)\right]$, where $E_{k}$ and $E_{K}$ are the unit groups of $k$ and $K$ respectively.

5.1. The number of classes capitulating in each $\mathbb{K}_{j}$.

Recall that $\kappa_{\mathbb{K}_{j}}$ denotes the capitulation kernel of the unramified extension $\mathbb{K}_{j} / \mathbb{k}_{\text {. }}$

Theorem 5.2. Let $\mathbb{K}_{j}, 1 \leq j \leq 3$, be the three unramified quadratic extensions of $\mathbb{k}$ defined above. Then

1. $\left|\kappa_{\mathbb{K}_{1}}\right|=4$.

2. Let $\epsilon_{p q_{2}}=a+b \sqrt{p q_{2}}$, then

i. If $a \pm 1$ is a square in $\mathbb{N}$ and $2 q_{1}(x+1), 2 q_{1}(x-1)$ are not, then $\left|\kappa_{\mathbb{K}_{2}}\right|=4$.

ii. In the other cases $\left|\kappa_{\mathbb{K}_{2}}\right|=2$.

3. Let $\epsilon_{p q_{1}}=a+b \sqrt{p q_{1}}$, then

i. If $a \pm 1$ is a square in $\mathbb{N}$ and $2 q_{2}(x+1), 2 q_{2}(x-1)$ are not, then $\left|\kappa_{\mathbb{K}_{3}}\right|=4$.

ii. In the other cases $\left|\kappa_{\mathbb{K}_{3}}\right|=2$.

Proof. Note first that, according to Lemma 2.7 $x+1$ and $x-1$ are never squares in $\mathbb{N}$, hence by Lemma 2.6, $E_{\mathbb{k}}=\left\langle i, \epsilon_{p q_{1} q_{2}}\right\rangle$.

1. By Proposition 3.1 we have $E_{\mathbb{K}_{1}}=\left\langle i, \epsilon_{p}, \epsilon_{q_{1} q_{2}}, \sqrt{\epsilon_{p q_{1} q_{2}}}\right\rangle$ or $E_{\mathbb{K}_{1}}=\left\langle i, \epsilon_{p}, \epsilon_{q_{1} q_{2}}\right.$,

$\left.\sqrt{\epsilon_{q_{1} q_{2}} \epsilon_{p q_{1} q_{2}}}\right\rangle$, hence $N_{\mathbb{K}_{1} / \mathbb{k}_{\mathrm{k}}}\left(E_{\mathbb{K}_{1}}\right)=\left\langle-1, \epsilon_{p q_{1} q_{2}}\right\rangle$. Thus $\left[E_{\mathbb{k}}: N_{\mathbb{K}_{1} / \mathbb{k}_{\mathrm{k}}}\left(E_{\mathbb{K}_{1}}\right)\right]=2$. Therefore Theorem 5.1 implies that $\left|\kappa_{\mathbb{K}_{1}}\right|=4$.

2. i. If $a \pm 1$ is a square in $\mathbb{N}$ and $2 q_{1}(x+1), 2 q_{1}(x-1)$ are not, then Proposition [3.2(2)(i) yields that $N_{\mathbb{K}_{2} / \mathbb{k}}\left(E_{\mathbb{K}_{2}}\right)=\left\langle i, \epsilon_{p q_{1} q_{2}}^{2}\right\rangle$, hence $\left[E_{\mathbb{k}}: N_{\mathbb{K}_{2} / \mathbb{k}}\left(E_{\mathbb{K}_{2}}\right)\right]=2$. Thus Theorem 5.1 implies that $\left|\kappa_{\mathbb{K}_{2}}\right|=4$.

ii. The other cases are grouped together in Proposition 3.2 (assertions 1, 2), then $N_{\mathbb{K}_{2} / \mathbb{k}}\left(E_{\mathbb{K}_{2}}\right)=\left\langle i, \epsilon_{p q_{1} q_{2}}\right\rangle$. Thus $\left[E_{\mathbb{k}}: N_{\mathbb{K}_{2} / \mathbb{k}_{\mathrm{k}}}\left(E_{\mathbb{K}_{2}}\right)\right]=1$, and Theorem 5.1 implies that $\left|\kappa_{\mathbb{K}_{2}}\right|=2$.

3. This point is similarly treated.

\subsection{Capitulation in $\mathbb{K}_{1}$.}

Theorem 5.3. Let $p, q_{1}$ and $q_{2}$ be different primes such that $p \equiv-q_{1} \equiv-q_{2} \equiv 1$ $(\bmod 4)$. Put $\mathbb{k}=\mathbb{Q}\left(\sqrt{p q_{1} q_{2}}, i\right), \mathbb{K}_{1}=\mathbb{Q}\left(\sqrt{p}, \sqrt{q_{1} q_{2}}, i\right)$ and $\epsilon_{p q_{1} q_{2}}=x+y \sqrt{p q_{1} q_{2}}$, then 
1. If $2 p(x \pm 1)$ is a square in $\mathbb{N}$, then $\kappa_{\mathbb{K}_{1}}=\left\langle\left[\mathcal{H}_{1}\right],\left[\mathcal{Q}_{1}\right]\right\rangle$.

2. Else, $\kappa_{\mathbb{K}_{1}}=\left\langle\left[\mathcal{H}_{1}\right],\left[\mathcal{H}_{2}\right]\right\rangle$.

Proof. We have already shown, in Lemma 4.1, that $\mathcal{H}_{1}, \mathcal{H}_{2}, \mathcal{Q}_{j}$ and $\mathcal{H}_{k} \mathcal{Q}_{j}, j, k=1,2$, are not principal in $\mathrm{k}$. On the other hand, by Proposition 6.3 of $[8] \mathcal{H}_{1}$ and $\mathcal{H}_{2}$ capitulate in $\mathbb{K}_{1}$.

1. If $2 p(x \pm 1)$ is a square in $\mathbb{N}$, then by [4, Proposition 2] $\mathcal{H}_{1} \mathcal{H}_{2}$ is principal in $\mathbb{k}$, i.e. $\left[\mathcal{H}_{1}\right]=\left[\mathcal{H}_{2}\right]$. The proof of the Proposition 3.1, allows us to conclude that $q_{1} \epsilon_{q_{1} q_{2}}$ and $q_{2} \epsilon_{q_{1} q_{2}}$ are squares in $\mathbb{K}_{1}$; hence there exists $\gamma \in \mathbb{K}_{1}$ such that $\mathcal{Q}_{1}^{2}=\left(\gamma^{2}\right)$. Thus $\mathcal{Q}_{1}=(\gamma)$, so the result.

2. If $2 p(x+1)$ and $2 p(x-1)$ are not squares in $\mathbb{N}$, then $\mathcal{H}_{1} \mathcal{H}_{2}$ is not principal in $\mathbb{k}$; which yields the result.

Numerical Examples 5.4.

1. The case where $2 p(x \pm 1)$ is a square in $\mathbb{N}$.

\begin{tabular}{|c|c|c|c|c|c|c|}
\hline$d=p . q_{1} . q_{2}$ & $2 p(x+1)$ & $2 p(x-1)$ & $\mathcal{H}_{1} \mathcal{H}_{2}$ in $\mathrm{k}$ & $\mathcal{Q}_{1}$ in $\mathrm{k}$ & $\mathcal{H}_{1}$ & $\mathcal{Q}_{1}$ \\
\hline $105=5.3 .7$ & 420 & $400=20^{2}$ & {$[0,0]$} & {$[2,0]$} & {$[0,0]$} & {$[0,0]$} \\
\hline $345=5.23 .3$ & 67620 & $67600=260^{2}$ & {$[0,0]$} & {$[2,0]$} & {$[0,0]$} & {$[0,0]$} \\
\hline $357=17.3 .7$ & 357 & $289=17^{2}$ & {$[0,0,0]$} & {$[1,1,0]$} & {$[0,0]$} & {$[0,0]$} \\
\hline $561=17.11 .3$ & 17774724 & $17774656=4216^{2}$ & {$[0,0,0]$} & {$[0,0,1]$} & {$[0,0,0]$} & {$[0,0,0]$} \\
\hline $645=5.3 .43$ & 645 & $625=25^{2}$ & {$[0,0]$} & {$[4,0]$} & {$[0,0]$} & {$[0,0]$} \\
\hline $705=5.47 .3$ & 2371620 & $2371600=1540^{2}$ & {$[0,0]$} & {$[6,0]$} & {$[0,0]$} & {$[0,0]$} \\
\hline $805=5.7 .23$ & 7245 & $7225=85^{2}$ & {$[0,0]$} & {$[4,0]$} & {$[0,0]$} & {$[0,0]$} \\
\hline
\end{tabular}

2. The case where $2 p(x+1)$ and $2 p(x-1)$ are not squares in $\mathbb{N}$.

\begin{tabular}{|c|c|c|c|c|c|}
\hline$d=p . q_{1} . q_{2}$ & $2 p(x+1)$ & $2 p(x-1)$ & $\mathcal{H}_{1} \mathcal{H}_{2}$ in $\mathrm{k}$ & $\mathcal{H}_{1}$ & $\mathcal{H}_{2}$ \\
\hline $165=5.3 .11$ & 75 & 55 & {$[2,0]$} & {$[0,0]$} & {$[0,0]$} \\
\hline $273=13.7 .3$ & 18928 & 18876 & {$[2,0]$} & {$[0,0]$} & {$[0,0]$} \\
\hline $285=5.3 .19$ & 95 & 75 & {$[4,0]$} & {$[0,0]$} & {$[0,0]$} \\
\hline $429=13.11 .3$ & 1911 & 1859 & {$[4,0]$} & {$[0,0]$} & {$[0,0]$} \\
\hline $465=5.3 .31$ & 158720 & 158700 & {$[4,0]$} & {$[0,0]$} & {$[0,0]$} \\
\hline $609=29.7 .3$ & 35130368 & 35130252 & {$[4,0]$} & {$[0,0]$} & {$[0,0]$} \\
\hline $665=5.7 .19$ & 137200 & 137180 & {$[6,0]$} & {$[0,0]$} & {$[0,0]$} \\
\hline $741=13.19 .3$ & 3211 & 3159 & {$[6,0]$} & {$[0,0]$} & {$[0,0]$} \\
\hline $1533=73.3 .7$ & 37303 & 37011 & {$[3,1,0]$} & {$[0,0,0]$} & {$[0,0,0]$} \\
\hline
\end{tabular}

\subsection{Capitulation in $\mathbb{K}_{2}$.}

Let $p, q_{1}$ and $q_{2}$ be different primes such that $p \equiv-q_{1} \equiv-q_{2} \equiv 1(\bmod 4)$. Put $\mathbb{K}_{\mathrm{k}}=\mathbb{Q}\left(\sqrt{p q_{1} q_{2}}, i\right), \mathbb{K}_{2}=\mathbb{Q}\left(\sqrt{q_{1}}, \sqrt{p q_{2}}, i\right)$ and $\epsilon_{p q_{2}}=a+b \sqrt{p q_{2}}$.

Lemma 5.5. If $a \pm 1$ is a square in $\mathbb{N}$, then $p \equiv 1(\bmod 8)$.

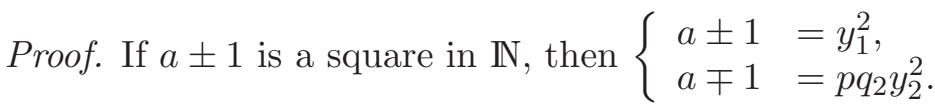

Hence $1=\left(\frac{a \pm 1}{p}\right)=\left(\frac{a \mp 1 \pm 2}{p}\right)=\left(\frac{2}{p}\right)$.

Therefore, if we suppose that $a \pm 1$ is a square in $\mathbb{N}$, then from Proposition 4.3 we get:

i. If $2 p(x \pm 1)$ is a square in $\mathbb{N}$, then $\operatorname{Am}_{s}(\mathbb{k} / \mathbb{Q}(i))=\left\langle\left[\mathcal{H}_{1}\right],\left[\mathcal{Q}_{1}\right]\right\rangle$. 
ii. Else, $\operatorname{Am}_{s}(\mathbb{k} / \mathbb{Q}(i))=\left\langle\left[\mathcal{H}_{1}\right],\left[\mathcal{H}_{2}\right]\right\rangle$.

iii. there exists an unambiguous ideal $\mathcal{I}$ in $\mathbb{k} / \mathbb{Q}(i)$ of order 2 such that

$$
\operatorname{Am}(\mathbb{k} / \mathbb{Q}(i))= \begin{cases}\left\langle\left[\mathcal{H}_{1}\right],\left[\mathcal{Q}_{1}\right],[\mathcal{I}]\right\rangle, & \text { if } 2 p(x \pm 1) \text { is a square in } \mathbb{N}, \\ \left\langle\left[\mathcal{H}_{1}\right],\left[\mathcal{H}_{2}\right],[\mathcal{I}]\right\rangle, & \text { otherwise. }\end{cases}
$$

The ideal $\mathcal{I}$ can be constructed by using the result:

Lemma 5.6 ([19]). Let $p_{1}, p_{2}, \ldots, p_{n}$ be distinct primes and for each $j$, let $e_{j}= \pm 1$. Then there exist infinitely many primes $\ell$ such that $\left(\frac{p_{j}}{\ell}\right)=e_{j}$, for all $j$.

Let $\ell$ be a prime congruent to $1(\bmod 4)$ and satisfying $\left(\frac{p q_{1} q_{2}}{\ell}\right)=-\left(\frac{q_{1}}{\ell}\right)=1$, thus $\ell$ splits completely in $\mathbb{k}$. Therefore $\mathcal{I}$ is one of the ideals of $\mathbb{k}$ above $\ell$; since $\left(\frac{q_{1}}{\ell}\right)=-1$, so $\mathcal{I}$ remaind inert in $\mathbb{K}_{2}$. We proceed as in [7] to prove that $\mathcal{I}, \mathcal{H}_{1} \mathcal{I}, \mathcal{H}_{2} \mathcal{I}$ and $\mathcal{H}_{1} \mathcal{H}_{2} \mathcal{I}$ or $\mathcal{I}, \mathcal{H}_{1} \mathcal{I}, \mathcal{Q}_{1} \mathcal{I}$ and $\mathcal{Q}_{1} \mathcal{H}_{1} \mathcal{I}$ are not principal in $\mathrm{k}$.

Theorem 5.7. Keep the previous hypothesis and notations and put $\epsilon_{p q_{2}}=a+b \sqrt{p q_{2}}$, $\epsilon_{p q_{1} q_{2}}=x+y \sqrt{p q_{1} q_{2}}$.

1. If $a \pm 1$ is a square in $\mathbb{N}$ and $2 q_{1}(x+1), 2 q_{1}(x-1)$ are not, then $\kappa_{\mathbb{K}_{2}}=\left\langle\left[\mathcal{Q}_{1}\right],[\mathcal{I}]\right\rangle$ or $\left\langle\left[\mathcal{Q}_{1}\right],\left[\mathcal{H}_{1} \mathcal{I}\right]\right\rangle$.

2. If $a \pm 1$ and $2 q_{1}(x \pm 1)$ are squares in $\mathbb{N}$, then $\kappa_{\mathbb{K}_{2}}=\langle[\mathcal{I}]\rangle$ or $\left\langle\left[\mathcal{I} \mathcal{H}_{1}\right]\right\rangle$ or $\left\langle\left[\mathcal{I} \mathcal{H}_{2}\right]\right\rangle$ or $\left\langle\left[\mathcal{I H}_{1} \mathcal{H}_{2}\right]\right\rangle$.

3. If $a+1$ and $a-1$ are not squares in $\mathbb{N}$ and $2 q_{1}(x \pm 1)$ is, then $\kappa_{\mathbb{K}_{2}}=\left\langle\left[\mathcal{Q}_{2}\right]\right\rangle=\left\langle\left[\mathcal{H}_{1} \mathcal{H}_{2}\right]\right\rangle$. 4. If $a+1, a-1,2 q_{1}(x+1)$ and $2 q_{1}(x-1)$ are not squares in $\mathbb{N}$, then $\kappa_{\mathbb{K}_{2}}=\left\langle\left[\mathcal{Q}_{1}\right]\right\rangle$.

Proof. Let $\mathcal{H}_{1}, \mathcal{H}_{2}, \mathcal{Q}_{1}$ and $\mathcal{Q}_{2}$ denote always the ideals of $\mathrm{k}$ above $\pi_{1}=e+2 i f$, $\pi_{2}=e-2 i f, q_{1}$ and $q_{2}$ respectively.

1. Suppose $a \pm 1$ is a square in $\mathbb{N}$ and $2 q_{1}(x+1), 2 q_{1}(x-1)$ are not. We know according to Proposition 3.2 that $E_{\mathbb{K}_{2}}=\left\langle i, \sqrt{\epsilon_{q_{1}} \epsilon_{p q_{2}}}, \epsilon_{p q_{1} q_{2}}, \sqrt{i \epsilon_{q_{1}}}\right\rangle$ and that four classes capitulate in $\mathbb{K}_{2}$ one of them is $\mathcal{Q}_{1}$. To proof the result, it suffices to prove that $\mathcal{H}_{1}$ does not capitulate in $\mathbb{K}_{2}$.

If $\mathcal{H}_{1}$ capitulates in $\mathbb{K}_{2}$, then there exists $\alpha \in \mathbb{K}_{2}$ such that $\mathcal{H}_{1}=(\alpha)$; hence $\left(\alpha^{2}\right)=$ $\left(\pi_{1}\right)$. As a result, there exists a unit $\epsilon \in \mathbb{K}_{2}$ such that $\pi_{1} \epsilon=\alpha^{2}$. The unit $\epsilon$ can not be real or purely imaginary. In fact, if it is real (same proof if it is purely imaginary), then by putting $\alpha=\alpha_{1}+i \alpha_{2}$, where $\alpha_{i}$ are in $\mathbb{K}_{2}^{+}$, we get $\alpha_{1}^{2}-\alpha_{2}^{2}+2 \alpha_{1} \alpha_{2}=\epsilon(e+2 i f)$, thus

$$
\begin{cases}\alpha_{1}^{2}-\alpha_{2}^{2} & =e \epsilon \\ \alpha_{1} \alpha_{2} & =f \epsilon\end{cases}
$$

hence $f \alpha_{1}^{2}-e \alpha_{2} \alpha_{1}-f \alpha_{2}^{2}=0$. But this implies that $\alpha_{1}=\frac{\alpha_{2}(e \pm \sqrt{p})}{f}$, and thus $\sqrt{p} \in \mathbb{K}_{2}^{+}$, which is absurd.

As $\pi_{1} \epsilon=\alpha^{2}$, so, by the norm $N_{\mathbb{K}_{2} / \mathbb{k}}$, we get $\pi_{1}^{2} N_{\mathbb{K}_{2} / \mathbb{k}}(\epsilon)=N_{\mathbb{K}_{2} / \mathbb{k}}(\alpha)^{2}$ with $N_{\mathbb{K}_{2} / \mathbb{k}}(\epsilon) \epsilon$ $E_{\mathrm{k}}=\left\langle i, \epsilon_{p q_{1} q_{2}}\right\rangle$. Therefore, we have the following result

$$
N_{\mathbb{K}_{2} / \mathbb{k}}(\epsilon) \in\left\{ \pm 1, \pm i, \pm \epsilon_{p q_{1} q_{2}}, \pm i \epsilon_{p q_{1} q_{2}}\right\} .
$$

a. If $N_{\mathbb{K}_{2} / \mathbb{k}}(\epsilon)= \pm i$, then $\pi_{1}^{2}( \pm i)=N_{\mathbb{K}_{2} / \mathbb{k}}(\alpha)^{2}$; hence $\sqrt{i} \in \mathbb{k}$, which is absurd.

b. If $N_{\mathbb{K}_{2} / \mathbb{k}}(\epsilon)= \pm \epsilon_{p q_{1} q_{2}}$, then $\pi_{1}^{2}\left( \pm \epsilon_{p q_{1} q_{2}}\right)=N_{\mathbb{K}_{2} / \mathbb{k}}(\alpha)^{2}$; this in turn yields that $\sqrt{\epsilon_{p q_{1} q_{2}}} \in \mathbb{k}$, which is absurd.

c. If $N_{\mathbb{K}_{2} / \mathbb{k}}(\epsilon)= \pm i \epsilon_{p q_{1} q_{2}}$, then $\pi_{1}^{2}\left( \pm i \epsilon_{p q_{1} q_{2}}\right)=N_{\mathbb{K}_{2} / \mathbb{k}}(\alpha)^{2}$; this in turn yields that $\sqrt{i \epsilon_{p q_{1} q_{2}}} \in \mathbb{k}$, which is absurd. 
d. If $N_{\mathbb{K}_{2} / \mathbb{k}}(\epsilon)=1$, then there exist $a, b, c$ and $d$ in $\{0,1\}$ such that $\epsilon=i^{a} \sqrt{\epsilon_{q_{1}} \epsilon_{p q_{2}}} b \epsilon_{p q_{1} q_{2}}^{c} \sqrt{i \epsilon_{q_{1}}} d$ and $N_{\mathbb{K}_{2} / \mathbb{k}}(\epsilon)=1$, hence $(-1)^{a} \epsilon_{p q_{1} q_{2}}^{2 c} i^{d}=1$. Thus obviously we must have $a=c=$ $d=0$. As a result, we get $\epsilon={\sqrt{\epsilon_{q_{1}} \epsilon_{p q_{2}}}}^{b}$ is a real, which is absurd.

e. If $N_{\mathbb{K}_{2} / \mathbb{k}}(\epsilon)=-1$, then, by applying the same argument, we get $\epsilon=i \sqrt{\epsilon_{q_{1}} \epsilon_{p q_{2}}}$, which is purely imaginary, and this is absurd.

To complete the proof of the first point of the corollary, we give examples that affirm the two cases of capitulation:

Numerical Examples 5.8.

$a \pm 1$ is a square in $\mathbb{N}$ and $2 q_{1}(x+1), 2 q_{1}(x-1)$ are not.

\begin{tabular}{|c|c|c|c|c|}
\hline$d=p . q_{1} . q_{2}$ & $\mathcal{I}$ in $\mathrm{k}$ & $\mathcal{H}_{1}$ & $\mathcal{I}$ in $\mathbb{K}_{2}$ & $\mathcal{H}_{1} \mathcal{I}$ in $\mathbb{K}_{2}$ \\
\hline $4029=17.3 .79$ & {$[5,1,1]$} & {$[170,0]$} & {$[170,0]$} & {$[0,0]$} \\
\hline $4029=17.79 .3$ & {$[5,0,0]$} & {$[30,0]$} & {$[30,0]$} & {$[0,0]$} \\
\hline $4029=17.79 .3$ & {$[0,0,1]$} & {$[30,0]$} & {$[30,0]$} & {$[0,0]$} \\
\hline $4029=17.79 .3$ & {$[5,0,0]$} & {$[30,0]$} & {$[30,0]$} & {$[0,0]$} \\
\hline $4029=17.79 .3$ & {$[5,0,0]$} & {$[30,0]$} & {$[30,0]$} & {$[0,0]$} \\
\hline $4029=17.79 .3$ & {$[0,1,1]$} & {$[30,0]$} & {$[30,0]$} & {$[0,0]$} \\
\hline $4029=17.79 .3$ & {$[0,0,1]$} & {$[30,0]$} & {$[30,0]$} & {$[0,0]$} \\
\hline $4029=17.79 .3$ & {$[5,1,0]$} & {$[30,0]$} & {$[30,0]$} & {$[0,0]$} \\
\hline
\end{tabular}

2. Suppose $a \pm 1$ and $2 q_{1}(x \pm 1)$ are squares in $\mathbb{N}$; then according to Proposition 3.2 , $2 p(x+1), 2 p(x-1), 2 q_{2}(x+1)$ and $2 q_{2}(x-1)$ are not squares in $\mathbb{N}$; but $2 p q_{2}(x \pm 1)$ is. Therefore [4, Proposition 2] implies that $\mathcal{H}_{1} \mathcal{H}_{2}$ and $\mathcal{Q}_{2}$ are not principal in $\mathbb{k}$, but $\mathcal{Q}_{1}$ and $\mathcal{H}_{1} \mathcal{H}_{2} \mathcal{Q}_{2}$ are; hence $\left[\mathcal{Q}_{2}\right]=\left[\mathcal{H}_{1} \mathcal{H}_{2}\right]$. Which implies that $\operatorname{Am}(\mathbb{k} / \mathbb{Q}(i))=\left\langle\left[\mathcal{H}_{1}\right],\left[\mathcal{H}_{2}\right],[\mathcal{I}]\right\rangle$. By using the same method applied in the above point, we show that $\mathcal{H}_{1}, \mathcal{H}_{2}$ and $\left[\mathcal{Q}_{2}\right]=\left[\mathcal{H}_{1} \mathcal{H}_{2}\right]$ do not capitulate in $\mathbb{K}_{2}$. Thus $\kappa_{\mathbb{K}_{2}}$ consists of one of the following ideal classes: $\mathcal{I}, \mathcal{H}_{1} \mathcal{I}, \mathcal{H}_{2} \mathcal{I}$ and $\mathcal{H}_{1} \mathcal{H}_{2} \mathcal{I}$. The following examples highlight these statements:

Numerical Examples 5.9.

$a \pm 1$ and $2 q_{1}(x \pm 1)$ are squares in $\mathbb{N}$.

\begin{tabular}{|c|c|c|c|c|}
\hline$d=p . q_{1} \cdot q_{2}$ & $\mathcal{I}$ in $\mathbb{K}_{2}$ & $\mathcal{I H}_{1}$ in $\mathbb{K}_{2}$ & $\mathcal{I} \mathcal{H}_{2}$ in $\mathbb{K}_{2}$ & $\mathcal{H}_{1} \mathcal{H}_{2} \mathcal{I}$ in $\mathbb{K}_{2}$ \\
\hline $969=17.19 .3$ & {$[0,0,0,0]$} & {$[3,1,1,1]$} & {$[0,1,1,0]$} & {$[0,1,0,0]$} \\
\hline $1533=73.3 .7$ & {$[0,0,1,0]$} & {$[0,0,0,0]$} & {$[21,1,0,1]$} & {$[21,1,0,0]$} \\
\hline $2037=97.3 .7$ & {$[9,0,0,0]$} & {$[9,0,0,1]$} & {$[0,0,0,0]$} & {$[0,0,0,1]$} \\
\hline $2193=17.43 .3$ & {$[3,0,0,0]$} & {$[0,0,1,0]$} & {$[0,1,1,0]$} & {$[0,0,0,0]$} \\
\hline
\end{tabular}

3. Suppose $a+1$ and $a-1$ are not squares in $\mathbb{N}$, and assume $2 q_{1}(x \pm 1)$ is. Then Propositions 1 and 2 of [4] imply that $\mathcal{Q}_{1}$ is principal in $\mathbb{k}, \mathcal{Q}_{2}$ and $\mathcal{H}_{1} \mathcal{H}_{2}$ are not, and $\left[\mathcal{Q}_{2}\right]=\left[\mathcal{H}_{1} \mathcal{H}_{2}\right]$. Moreover, $p(a \pm 1)$ or $2 p(a \pm 1)$ is a square in $\mathbb{N}$, hence $q_{2} \epsilon_{p q_{2}}$ or $2 q_{2} \epsilon_{p q_{2}}$ is a square in $\mathbb{K}_{2}$; and this yields that $\mathcal{Q}_{2}$ and $\mathcal{H}_{1} \mathcal{H}_{2}$ capitulate in $\mathbb{K}_{2}$. Here are some examples that illustrate our results.

Numerical Examples 5.10.

$a+1$ and $a-1$ are not squares in $\mathbb{N}$ and $2 q_{1}(x \pm 1)$ is. 


\begin{tabular}{|c|c|c|c|c|c|c|c|}
\hline$d=p . q_{1} \cdot q_{2}$ & $a$ & $2 q_{1}(x+1)$ & $2 q_{1}(x-1)$ & $\begin{array}{c}\mathcal{H}_{1} \mathcal{H}_{2} \\
\text { in } \mathrm{k}\end{array}$ & $\begin{array}{c}\mathcal{Q}_{2} \\
\text { in } \mathrm{k}\end{array}$ & $\mathcal{H}_{1} \mathcal{H}_{2}$ & $\mathcal{Q}_{2}$ \\
\hline $165=5.11 .3$ & 4 & 165 & $121=11^{2}$ & {$[2,0]$} & {$[2,0]$} & {$[0,0,0]$} & {$[0,0,0]$} \\
\hline $273=13.3 .7$ & 1574 & 4368 & $4356=66^{2}$ & {$[2,0]$} & {$[2,0]$} & {$[0,0,0]$} & {$[0,0,0]$} \\
\hline $285=5.19 .3$ & 4 & $361=19^{2}$ & 285 & {$[4,0]$} & {$[4,0]$} & {$[0,0,0]$} & {$[0,0,0]$} \\
\hline $385=5.11 .7$ & 6 & $2108304=1452^{2}$ & 2108260 & {$[2,0]$} & {$[2,0]$} & {$[0,0,0]$} & {$[0,0,0]$} \\
\hline $429=13.3 .11$ & 12 & $441=21^{2}$ & 429 & {$[4,0]$} & {$[4,0]$} & {$[0,0,0]$} & {$[0,0,0]$} \\
\hline $465=5.31 .3$ & 4 & $984064=992^{2}$ & 983940 & {$[4,0]$} & {$[4,0]$} & {$[0,0,0]$} & {$[0,0,0]$} \\
\hline $609=29.7 .3$ & 28 & $8479744=2912^{2}$ & 8479716 & {$[4,0]$} & {$[4,0]$} & {$[0,0,0]$} & {$[0,0,0]$} \\
\hline $665=5.19 .7$ & 6 & 521360 & $521284=722^{2}$ & {$[6,0]$} & {$[6,0]$} & {$[0,0,0]$} & {$[0,0,0]$} \\
\hline $741=13.3 .19$ & 85292 & 741 & $729=27^{2}$ & {$[6,0]$} & {$[6,0]$} & {$[0,0,0]$} & {$[0,0,0]$} \\
\hline $777=37.7 .3$ & 295 & $3136=56^{2}$ & 3108 & {$[4,0]$} & {$[4,0]$} & {$[0,0,0]$} & {$[0,0,0]$} \\
\hline $885=5.59 .3$ & 4 & 14160 & $13924=118^{2}$ & {$[6,0]$} & {$[6,0]$} & {$[0,0,0]$} & {$[0,0,0]$} \\
\hline $897=13.3 .23$ & 415 & $3600=60^{2}$ & 3588 & {$[4,0]$} & {$[4,0]$} & {$[0,0,0]$} & {$[0,0,0]$} \\
\hline $1045=5.11 .19$ & 39 & $1089=33^{2}$ & 1045 & {$[4,0]$} & {$[4,0]$} & {$[0,0,0]$} & {$[0,0,0]$} \\
\hline
\end{tabular}

4. If $a+1, a-1,2 q_{1}(x+1)$ and $2 q_{1}(x-1)$ are not squares in $\mathbb{N}$, then $\mathcal{Q}_{1}$ is not principal in $\mathbb{k}$; and as $\sqrt{q_{1}} \in \mathbb{K}_{2}$, so $\mathcal{Q}_{1}$ capitulate in $\mathbb{K}_{2}$.

Numerical Examples 5.11.

$a+1, a-1,2 q_{1}(x+1)$ and $2 q_{1}(x-1)$ are not squares in $\mathbb{N}$.

\begin{tabular}{|c|c|c|c|c|c|c|}
\hline$d=p . q_{1} \cdot q_{2}$ & $a+1$ & $a-1$ & $2 q_{1}(x+1)$ & $2 q_{1}(x-1)$ & $\mathcal{Q}_{1}$ in $\mathbb{k}$ & $\mathcal{Q}_{1}$ \\
\hline $105=5.7 .3$ & 5 & 3 & 588 & 560 & {$[2,0]$} & {$[0,0]$} \\
\hline $165=5.3 .11$ & 90 & 88 & 45 & 33 & {$[2,0]$} & {$[0,0]$} \\
\hline $273=13.7 .3$ & 26 & 24 & 10192 & 10164 & {$[2,0]$} & {$[0,0]$} \\
\hline $285=5.3 .19$ & 40 & 38 & 57 & 45 & {$[4,0]$} & {$[0,0]$} \\
\hline $345=5.3 .23$ & 1127 & 1125 & 40572 & 40560 & {$[2,0]$} & {$[0,0]$} \\
\hline $345=5.23 .3$ & 5 & 3 & 311052 & 310960 & {$[2,0]$} & {$[0,0]$} \\
\hline $385=5.7 .11$ & 90 & 88 & 1341648 & 1341620 & {$[2,0]$} & {$[0,0]$} \\
\hline $429=13.11 .3$ & 26 & 24 & 1617 & 1573 & {$[4,0]$} & {$[0,0]$} \\
\hline $465=5.3 .31$ & 250 & 248 & 95232 & 95220 & {$[4,0]$} & {$[0,0]$} \\
\hline
\end{tabular}

5.4. Capitulation in $\mathbb{K}_{3}$.

Let $p, q_{1}$ and $q_{2}$ be different primes satisfying $p \equiv-q_{1} \equiv-q_{2} \equiv 1(\bmod 4)$. Put $\mathrm{k}=\mathbb{Q}\left(\sqrt{p q_{1} q_{2}}, i\right), \mathbb{K}_{3}=\mathbb{Q}\left(\sqrt{q_{2}}, \sqrt{p q_{1}}, i\right)$ and $\epsilon_{p q_{1}}=a+b \sqrt{p q_{1}}$. As $q_{1}$ and $q_{2}$ play symmetric roles, so the following results are deduced from the above by analogy. Let $\mathcal{I}$ be the ideal defined as above and assume the prime $\ell$ satisfies the conditions: $\ell \equiv 1$ $(\bmod 4)$ and $\left(\frac{p q_{1} q_{2}}{\ell}\right)=-\left(\frac{q_{2}}{\ell}\right)=1$.

Theorem 5.12. Keep the obvious notations and hypothesis. Put $\epsilon_{p q_{1}}=a+b \sqrt{p q_{1}}$, then 1. If $a \pm 1$ is a square in $\mathbb{N}$ and $2 q_{2}(x+1), 2 q_{2}(x-1)$ are not, then $\kappa_{\mathbb{K}_{3}}=\left\langle\left[\mathcal{Q}_{2}\right],[\mathcal{I}]\right\rangle$ or $\left\langle\left[\mathcal{Q}_{2}\right],\left[\mathcal{H}_{1} \mathcal{I}\right]\right\rangle$.

2. If $a \pm 1$ and $2 q_{2}(x \pm 1)$ are squares in $\mathbb{N}$, then $\kappa_{\mathbb{K}_{3}}=\langle[\mathcal{I}]\rangle$ or $\left\langle\left[\mathcal{I H}_{1}\right]\right\rangle$ or $\left\langle\left[\mathcal{I H}_{2}\right]\right\rangle$ or $\left\langle\left[\mathcal{I H}_{1} \mathcal{H}_{2}\right]\right\rangle$.

3. If $a+1$ and $a-1$ are not squares in $\mathbb{N}$ and $2 q_{2}(x \pm 1)$ is, then $\kappa_{\mathbb{K}_{3}}=\left\langle\left[\mathcal{Q}_{1}\right]\right\rangle$.

4. If $a+1, a-1,2 q_{2}(x+1)$ and $2 q_{2}(x-1)$ are not squares in $\mathbb{N}$, then $\kappa_{\mathbb{K}_{3}}=\left\langle\left[\mathcal{Q}_{2}\right]\right\rangle$. 


\subsection{Capitulation in $\mathbb{k}^{(*)}$.}

The following theorem is a simple deduction from Theorems 5.3, 5.7 and 5.12.

Theorem 5.13. Let $p, q_{1}$ and $q_{2}$ be different primes satisfying $p \equiv-q_{1} \equiv-q_{2} \equiv 1$ $(\bmod 4)$. Put $\mathbb{k}=\mathbb{Q}\left(\sqrt{p q_{1} q_{2}}, i\right)$ and denote by $\mathbb{k}^{(*)}$ its genus field. Let $\epsilon_{p q_{1} q_{2}}=x+$ $y \sqrt{p q_{1} q_{2}}$ be the fundamental unit of $\mathbb{Q}\left(\sqrt{p q_{1} q_{2}}\right)$.

1. Assume $p \equiv 1(\bmod 8)$, then there exists an unambiguous ideal $\mathcal{I}$ of $\mathbb{k} / \mathbb{Q}(i)$ of order 2 such that:

i. If $2 p(x \pm 1)$ is a square in $\mathbb{N}$, then $\left\langle\left[\mathcal{H}_{1}\right],\left[\mathcal{Q}_{1}\right],[\mathcal{I}]\right\rangle \subseteq \kappa_{\mathbb{k}(*)}$.

ii. Else, $\left\langle\left[\mathcal{H}_{1}\right],\left[\mathcal{H}_{2}\right],[\mathcal{I}]\right\rangle \subseteq \kappa_{\mathbb{k}(*)}$.

2. Assume $p \equiv 5(\bmod 8)$.

i. If $2 p(x \pm 1)$ is a square in $\mathbb{N}$, then $\left\langle\left[\mathcal{H}_{1}\right],\left[\mathcal{Q}_{1}\right]\right\rangle \subseteq \kappa_{\mathbb{k}(*)}$.

ii. Else, $\left\langle\left[\mathcal{H}_{1}\right],\left[\mathcal{H}_{2}\right]\right\rangle \subseteq \kappa_{\mathbb{K}}(*)$.

\section{Application}

Let $p \equiv-q_{1} \equiv-q_{2} \equiv 1(\bmod 4)$ be different primes such that $\mathbf{C} l_{2}(\mathbb{k})$ is of type $(2,2,2)$. According to $[3], \mathbf{C l}_{2}(\mathbb{k})$ is of type $(2,2,2)$ if and only if $p, q_{1}$ and $q_{2}$ satisfy the following two conditions:

A: $p \equiv-q_{1} \equiv-q_{2} \equiv 1(\bmod 4)$ and $\left(\frac{2}{p}\right)=\left(\frac{q_{1}}{q_{2}}\right)=-\left(\frac{q_{2}}{q_{1}}\right)=1$.

$\mathrm{B}$ : One of the following three conditions is satisfied:

$$
\begin{aligned}
& \text { (I): }\left(\frac{p}{q_{1}}\right)\left(\frac{p}{q_{2}}\right)=-1 \text { and }\left(\frac{2}{q_{1}}\right)=\left(\frac{2}{q_{2}}\right)=-1 . \\
& \text { (II): }\left(\frac{p}{q_{1}}\right)\left(\frac{p}{q_{2}}\right)=-1,\left(\frac{2}{q_{1}}\right)=1 \text { and }\left(\frac{2}{q_{2}}\right)=-1 . \\
& \text { (III): }\left(\frac{p}{q_{1}}\right)=\left(\frac{p}{q_{2}}\right)=-1 \text { and }\left(\frac{2}{q_{1}}\right)\left(\frac{2}{q_{2}}\right)=-1 .
\end{aligned}
$$

Remark 6.1. We keep the notations defined in [5, Definition 1], and we add the following definition assuming $p \equiv-q_{1} \equiv-q_{2} \equiv 1(\bmod 4)$ satisfying the condition A.

1. $p, q_{1}$ and $q_{2}$ are said of type $B(I I I)(1)$ if $\left(\frac{p}{q_{1}}\right)=\left(\frac{p}{q_{2}}\right)=-1$ and $-\left(\frac{2}{q_{1}}\right)=\left(\frac{2}{q_{2}}\right)=1$.

2. $p, q_{1}$ and $q_{2}$ are said of type $B(I I I)(2)$ if $\left(\frac{p}{q_{1}}\right)=\left(\frac{p}{q_{2}}\right)=-1$ and $\left(\frac{2}{q_{1}}\right)=-\left(\frac{2}{q_{2}}\right)=1$.

To continue we need the following results.

Lemma 6.2. Let $p \equiv-q_{1} \equiv-q_{2} \equiv 1(\bmod 4)$ be different primes satisfying the condition $A$, and put $\epsilon_{p q_{2}}=a+b \sqrt{p q_{2}}$.

1. If $p, q_{1}$ and $q_{2}$ are of type $B(I)$ or $B(I I)$, then $a+1$ is not a square in $\mathbb{N}$.

2. If $p, q_{1}$ and $q_{2}$ are of type $B(I)(1)$ or $B(I I)(1)$, then $p(a-1)$ and $2 p(a+1)$ are not squares in $\mathbb{N}$.

3. If $p, q_{1}$ and $q_{2}$ are of type $B(I)(2)$ or $B(I I)(2)$, then $p(a+1)$ and $2 p(a-1)$ are not squares in $\mathbb{N}$.

4. If $p, q_{1}$ and $q_{2}$ are of type $B(I I I)(1)$, then $a-1$ and $p(a+1)$ are not squares in $\mathbb{N}$.

5. If $p, q_{1}$ and $q_{2}$ are of type $B(I I I)(2)$, then $a+1$ and $p(a-1)$ are not squares in $\mathbb{N}$.

6. If $p, q_{1}$ and $q_{2}$ are of type $B(I I I)$, then $2 p(a+1)$ is not a square in $\mathbb{N}$.

Proof. We know that $N\left(\epsilon_{p q_{2}}\right)=1$, then $a^{2}-1=b^{2} p q_{2}$, hence by Lemma 2.4 and the decomposition uniqueness in $\mathbb{Z}$ there exist $b_{1}, b_{2}$ in $\mathbb{Z}$ such that: 


$$
\text { (1) }\left\{\begin{array} { l } 
{ a \pm 1 = b _ { 1 } ^ { 2 } , } \\
{ a \mp 1 = p q _ { 2 } b _ { 2 } ^ { 2 } ; }
\end{array} \text { or (2) } \left\{\begin{array} { l } 
{ a \pm 1 = p b _ { 1 } ^ { 2 } , } \\
{ a \mp 1 = q _ { 2 } b _ { 2 } ^ { 2 } ; }
\end{array} \text { or } ( 3 ) \left\{\begin{array}{l}
a \pm 1=2 p b_{1}^{2}, \\
a \mp 1=2 q_{2} b_{2}^{2} ;
\end{array}\right.\right.\right.
$$

1. Suppose

$$
\left\{\begin{array}{l}
a+1=b_{1}^{2}, \\
a-1=p q_{2} b_{2}^{2},
\end{array}\right.
$$

then $\left(\frac{2}{q_{2}}\right)=1$, but this contradicts the conditions $B(I)$ and $B(I I)$, hence the result.

The other cases are checked similarly.

Remark 6.3. If $\mathbf{C} l_{2}(\mathbb{k})$ is of type $(2,2,2)$, then by Proposition 4.3 and [5, Lemma 3], we deduce that:

1. $\operatorname{Am}_{s}(\mathbb{k} / \mathbb{Q}(i))=\left\langle\left[\mathcal{H}_{1}\right],\left[\mathcal{Q}_{1}\right]\right\rangle \varsubsetneqq \operatorname{Am}(\mathbb{k} / \mathbb{Q}(i))=\mathrm{Cl}_{2}(\mathbb{k})=\left\langle\left[\mathcal{H}_{1}\right],\left[\mathcal{Q}_{1}\right],[\mathcal{I}]\right\rangle$, if $p, q_{1}$ and $q_{2}$ are of type $B(I I I)$,

2. $\operatorname{Am}_{s}(\mathbb{k} / \mathbb{Q}(i))=\left\langle\left[\mathcal{H}_{1}\right],\left[\mathcal{H}_{2}\right]\right\rangle \varsubsetneqq \operatorname{Am}(\mathbb{k} / \mathbb{Q}(i))=\mathrm{Cl}_{2}(\mathbb{k})=\left\langle\left[\mathcal{H}_{1}\right],\left[\mathcal{H}_{2}\right],[\mathcal{I}]\right\rangle$, otherwise.

Theorem 6.4. Let $p \equiv-q_{1} \equiv-q_{2} \equiv 1(\bmod 4)$ be different primes such that $\mathbf{C l}_{2}(\mathbb{k})$ is of type $(2,2,2)$, where $\mathbb{k}=\mathbb{Q}\left(\sqrt{p q_{1} q_{2}}, i\right)$.

1. Exactly four classes of $\mathbf{C l}_{2}(\mathbb{k})$ capitulate in $\mathbb{K}_{1}$.

i. If $p, q_{1}$ and $q_{2}$ are of type $B(I I I)$, then $\kappa_{\mathbb{K}_{1}}=\left\langle\left[\mathcal{H}_{1}\right],\left[\mathcal{Q}_{1}\right]\right\rangle$.

ii. Else, $\kappa_{\mathbb{K}_{1}}=\left\langle\left[\mathcal{H}_{1}\right],\left[\mathcal{H}_{2}\right]\right\rangle$.

2. Put $\epsilon_{p q_{2}}=a+b \sqrt{p q_{2}}$, then the capitulation in $\mathbb{K}_{2}$ is given by:

i. If $p, q_{1}$ and $q_{2}$ are of type $B(I)(1)$ or $B(I I)(1)$, then $\kappa_{\mathbb{K}_{2}}=\langle[\mathcal{I}]\rangle$ or $\left\langle\left[\mathcal{H}_{1} \mathcal{I}\right]\right\rangle$ or $\left\langle\left[\mathcal{H}_{2} \mathcal{I}\right]\right\rangle$ or $\left\langle\left[\mathcal{H}_{1} \mathcal{H}_{2} \mathcal{I}\right]\right\rangle$.

ii. If $p, q_{1}$ and $q_{2}$ are of type $B(I)(2)$ or $B(I I)(2)$, then

a. If $a-1$ is a square in $\mathbb{N}$, then $\kappa_{\mathbb{K}_{2}}=\left\langle\left[\mathcal{H}_{1} \mathcal{H}_{2}\right],[\mathcal{I}]\right\rangle$ or $\left\langle\left[\mathcal{H}_{1} \mathcal{H}_{2}\right],\left[\mathcal{H}_{1} \mathcal{I}\right]\right\rangle$.

b. Else, $\kappa_{\mathbb{K}_{2}}=\left\langle\left[\mathcal{Q}_{1}\right]\right\rangle=\left\langle\left[\mathcal{H}_{1} \mathcal{H}_{2}\right]\right\rangle$.

iii. If $p, q_{1}$ and $q_{2}$ are of type $B(I I I)$, then

a. If $a \pm 1$ is a square in $\mathbb{N}$, then $\kappa_{\mathbb{K}_{2}}=\left\langle\left[\mathcal{Q}_{1}\right],[\mathcal{I}]\right\rangle$ or $\left\langle\left[\mathcal{Q}_{1}\right],\left[\mathcal{H}_{1} \mathcal{I}\right]\right\rangle$.

b. Else, $\kappa_{\mathbb{K}_{2}}=\left\langle\left[\mathcal{Q}_{1}\right]\right\rangle$.

3. Put $\epsilon_{p q_{1}}=a+b \sqrt{p q_{1}}$, then the capitulation in $\mathbb{K}_{3}$ is given by:

i. If $p, q_{1}$ and $q_{2}$ are of type $B(I)(2)$ ou $B(I I)(2)$, then $\kappa_{\mathbb{K}_{3}}=\langle[\mathcal{I}]\rangle$ or $\left\langle\left[\mathcal{H}_{1} \mathcal{I}\right]\right\rangle$ or $\left\langle\left[\mathcal{H}_{2} \mathcal{I}\right]\right\rangle$ or $\left\langle\left[\mathcal{H}_{1} \mathcal{H}_{2} \mathcal{I}\right]\right\rangle$.

ii. If $p, q_{1}$ and $q_{2}$ are of type $B(I)(1)$ or $B(I I)(1)$, then

a. If $a-1$ is a square in $\mathbb{N}$, then $\kappa_{\mathbb{K}_{3}}=\left\langle\left[\mathcal{H}_{1} \mathcal{H}_{2}\right],[\mathcal{I}]\right\rangle$ or $\left\langle\left[\mathcal{H}_{1} \mathcal{H}_{2}\right],\left[\mathcal{H}_{1} \mathcal{I}\right]\right\rangle$.

b. Else, $\kappa_{\mathbb{K}_{3}}=\left\langle\left[\mathcal{Q}_{2}\right]\right\rangle=\left\langle\left[\mathcal{H}_{1} \mathcal{H}_{2}\right]\right\rangle$.

iii. If $p, q_{1}$ and $q_{2}$ are of type $B(I I I)$, then

a. If $a \pm 1$ is a square in $\mathbb{N}$, then $\kappa_{\mathbb{K}_{3}}=\left\langle\left[\mathcal{Q}_{2}\right],[\mathcal{I}]\right\rangle$ or $\left\langle\left[\mathcal{Q}_{2}\right],\left[\mathcal{H}_{1} \mathcal{I}\right]\right\rangle$.

b. Else, $\kappa_{\mathbb{K}_{2}}=\left\langle\left[\mathcal{Q}_{2}\right]\right\rangle$.

Proof. Let $\epsilon_{p q_{1} q_{2}}=x+y \sqrt{p q_{1} q_{2}}$ denote the fundamental unit of $\mathbb{Q}\left(\sqrt{p q_{1} q_{2}}\right)$.

1. We know, by [5. Lemma 3], that if $p, q_{1}$ and $q_{2}$ are of type $B(I I I)$, then $2 p(x-1)$ is a square in $\mathbb{N}$, and otherwise $2 p(x-1), 2 p(x+1)$ are not squares in $\mathbb{N}$. Thus Theorem 5.3 implies the results.

2. Put $\epsilon_{p q_{2}}=a+b \sqrt{p q_{2}}$.

i. Suppose $p, q_{1}$ and $q_{2}$ satisfy the conditions $A$ and $B(I)(1)$ or $B(I I)(1)$, then, by [5, Lemma 3], $2 q_{1}(x+1)$ is a square in $\mathbb{N}$. On the other hand, from Lemma 
6.2 $p(a-1)$ and $2 p(a+1)$ are not squares in $\mathbb{N}$, thus $a-1$ is a square in $\mathbb{N}$. Therefore, we are in the hypotheses of Theorem 5.7(2), thus the results.

ii. Suppose $p, q_{1}$ and $q_{2}$ satisfy the conditions $A$ and $B(I)(2)$ or $B(I I)(2)$, then, by [5. Lemma 3], $2 q_{2}(x-1)$ is a square in $\mathbb{N}$ i.e. $2 p q_{1}(x+1)$ is a square in $\mathbb{N}$. Thus [4. Proposition 1] implies that $\left[\mathcal{H}_{1} \mathcal{H}_{2}\right]=\left[Q_{1}\right]$. On the other hand, from Lemma 6.2. one of the numbers $a-1, p(a-1)$ or $2 p(a+1)$ is a square in $\mathbb{N}$. So we are in the hypotheses of Theorem 5.7 (1) or (4), thus the results.

iii. Suppose $p, q_{1}$ and $q_{2}$ satisfy the conditions $A$ and $B(I I I)$, then, by [5. Lemma 3], $2 p(x-1)$ is a square in $\mathbb{N}$, and by Lemma 6.2, $2 p(a+1)$ is not a square in $\mathbb{N}$. - If $p, q_{1}$ and $q_{2}$ are of type $B(I I I)(1)$, then Lemma 6.2 implies that one of the numbers $a+1, p(a-1)$ or $2 p(a-1)$ is a square in $\mathbb{N}$.

- If $p, q_{1}$ and $q_{2}$ are of type $B(I I I)(2)$, then Lemma 6.2 implies that one of the numbers $a-1, p(a+1)$ or $2 p(a-1)$ is a square in $\mathbb{N}$.

Therefore,

a. If $a \pm 1$ is a square in $\mathbb{N}$, then the result is assured by Theorem 5.7(1).

b. Else, the result is assured by Theorem 5.7(4).

3. These results are shown as in 2 .

Corollary 6.5. Keep the hypotheses and notations mentioned in Theorem 6.4. Then all the classes of $\mathrm{Cl}_{2}(\mathrm{k})$ capitulate in $\mathbb{k}^{(*)}$ i.e.

$$
\kappa_{\mathbb{k}(*)}=\mathbf{C} l_{2}(\mathbb{k})=\operatorname{Am}(\mathbb{k} / \mathbb{Q}(i)) .
$$

\section{Acknowledgement}

We would like to thank the referee of our paper for his precious remarks and suggestions.

\section{REFERENCES}

[1] A. Azizi, Sur la capitulation des 2-classes d'idéaux de $\mathrm{k}=\mathbb{Q}(\sqrt{2 p q}, i)$, où $p \equiv-q \equiv 1(\bmod 4)$, Acta. Arith. 94 (2000), 383-399, Zbl 0953.11033, MR 1779950.

[2] A. Azizi, Unités de certains corps de nombres imaginaires et abéliens sur $\mathbb{Q}$, Ann. Sci. Math. Québec 23 (1999), no 1, 15-21, Zbl 1041.11072, MR 1721726.

[3] A. Azizi and M. Taous, Détermination des corps $\mathbf{k}=\mathbb{Q}(\sqrt{d}, \sqrt{-1})$ dont les 2-groupes de classes sont de type $(2,4)$ ou $(2,2,2)$, Rend. Istit. Mat. Univ. Trieste. 40 (2008), 93-116, Zbl 1215.11107, MR2583453.

[4] A. Azizi, A. Zekhnini and M. Taous, On the generators of the 2-class group of the field $\mathbb{Q}(\sqrt{d}, i)$, IJPAM, Volume 81, No. 5 (2012), 773-784.

[5] A. Zekhnini, A. Azizi and M. Taous, On the generators of the 2 -class group of the field $\mathbb{Q}\left(\sqrt{q_{1} q_{2} p}, i\right)$ Correction to Theorem 3 of [5], IJPAM, Volume 103, No. 1 (2015), 99-107.

[6] A. Azizi, A. Zekhnini and M. Taous, On the strongly ambiguous classes of $\mathbb{k} / \mathbb{Q}(i)$ where $\mathbb{k}=$ $\mathbb{Q}\left(\sqrt{2 p_{1} p_{2}}, i\right)$, Asian-Eur. J. Math. 7 (2014), no. 1, Zbl 1292.11119, MR3189588.

[7] A. Azizi, A. Zekhnini and M. Taous, On the strongly ambiguous classes of some biquadratic number fields, to appear in Mathematica Bohemica.

[8] A. Azizi, A. Zekhnini and M. Taous, Capitulation in the absolutely abelian extensions of some fields $\mathbb{Q}\left(\sqrt{p_{1} p_{2} q}, \sqrt{-1}\right)$, http://arxiv.org/abs/1507.00295v1 submitted.

[9] A. Azizi, A. Zekhnini and M. Taous, Structure of $\mathrm{Gal}\left(\mathrm{k}_{2}^{(2)} / \mathrm{k}\right)$ for some fields $\mathrm{k}=\mathbb{Q}\left(\sqrt{2 p_{1} p_{2}}, i\right)$ with $\mathbf{C l}_{2}(\mathbb{k}) \simeq(2,2,2)$, Abh. Math. Sem. Univ. Hamburg, Vol 84, 2 (2014), 203-231, MR3267742. 
[10] A. Azizi, A. Zekhnini, M. Taous and Daniel C. Mayer, Principalization of 2-class groups of type $(2,2,2)$ of biquadratic fields $\mathbb{Q}\left(\sqrt{p_{1} p_{2} q}, i\right)$, Int. J. Number Theory, Vol. 11, 4, (2015) 1177-1215.

[11] A. Azizi, A. Zekhnini and M. Taous, Coclass of $\operatorname{Gal}\left(\mathbb{k}_{2}^{(2)} / \mathbb{k}\right)$ for some fields $\mathbb{k}=$ $\mathbb{Q}\left(\sqrt{p_{1} p_{2} q}, i\right)$ with 2 -class groups of type $(2,2,2)$, J. Algebra Appl, Vol. 15, No. 2 (2016) DOI: 10.1142/S0219498816500274.

[12] C. Chevalley, Sur la théorie du corps de classes dans les corps finis et les corps locaux, J. Fac. Sc. Tokyo, Sect. 1, t.2, (1933), 365-476, Zbl 0008.05301.

[13] F. Lemmermeyer, The ambiguous class number formula revisited, J. of the Ramanujan Math. Soc. Volume 28, 4, 415-421, MR3158989.

[14] F. P. Heider and B. Schmithals, Zur kapitulation der idealklassen in unverzweigten primzyklischen erweiterungen, J. Reine Angew. Math. 366 (1982), 1-25, Zbl 0505.12016, MR 0671319.

[15] F. Terada, A principal ideal theorem in the genus fields, Tohoku Math. J. 23, No. 2 (1971), 697-718, Zbl0243.12003, MR0306158.

[16] H. Furuya, Principal ideal theorems in the genus field for absolutely abelian extensions, J. Number Theory 9, (1977), 4-15, Zbl 0347.12006, MR0429820.

[17] H. Hasse, Über die Klassenzahl abelscher Zahlkörper, Berlin, Akademie-Verlag, (1952), Zbl46.260, MR 0049239.

[18] H. Wada, On the class number and the unit group of certain algebraic number fields, J. Fac. Univ. Tokyo Sect. I 13 (1966), 201-209, Zbl 0158.30103, MR 0214565.

[19] P. J. Sime, On the ideal class group of real biquadratic fields, Trans. Am. Math. Soc. 347 No. 12 (1995), 4855-4876.

[20] S. Louboutin. Hasse unit indices of dihedral octic CM-fields, Math. Nachr. 215 (2000), 107-113, Zbl 0972.11105, MR 1768197.

[21] T. Kubota, Über den bizyklischen biquadratischen Zahlkörper, Nagoya Math. J. 10 (1956), 65-85, Zbl 0074.03001, MR 0083009.

[22] T. M. McCall, C. J. Parry and R. R. Ranalli, Imaginary bicyclic biquadratic fields with cyclic 2-class group, J. Number Theory 53, 88-99 (1995), Zbl 0831.11059, MR 1344833.

Abdelmalek Azizi: Mohammed First University, Mathematics Department, Sciences FaCULTY, Oujda, Morocco

E-mail address: abdelmalekazizi@yahoo.fr

Abdelkader Zekhnini: Mohammed First University, Mathematics Department, PluridisCIPLINARY FACULTY, NADOR, MOROCCO

E-mail address: zekha1@yahoo.fr

Mohammed Taous: Moulay Ismail University, Mathematics Department, Sciences and Techniques Faculty, Errachidia, Morocco.

E-mail address: taousm@hotmail.com 